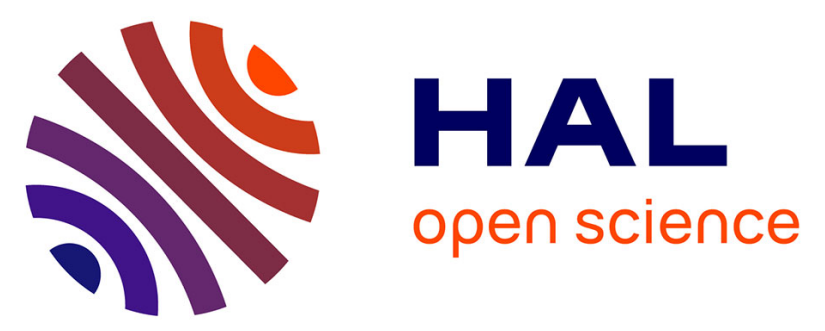

\title{
The human NANOS3 gene contributes to lung tumour invasion by inducing epithelial-mesenchymal transition
}

Simon Grelet, Vanessa Andries, Myriam Polette, Christine Gilles, Katrien Staes, Anne-Pascaline Martin, Claire Kileztky, Christine Terryn, Véronique Dalstein, Chun-Wen Cheng, et al.

\section{To cite this version:}

Simon Grelet, Vanessa Andries, Myriam Polette, Christine Gilles, Katrien Staes, et al.. The human NANOS3 gene contributes to lung tumour invasion by inducing epithelial-mesenchymal transition. Journal of Pathology, 2015, 237 (1), pp.25-37. 10.1002/path.4549 . hal-03007236

\section{HAL Id: hal-03007236 \\ https://hal.univ-reims.fr/hal-03007236}

Submitted on 26 Nov 2020

HAL is a multi-disciplinary open access archive for the deposit and dissemination of scientific research documents, whether they are published or not. The documents may come from teaching and research institutions in France or abroad, or from public or private research centers.
L'archive ouverte pluridisciplinaire HAL, est destinée au dépôt et à la diffusion de documents scientifiques de niveau recherche, publiés ou non, émanant des établissements d'enseignement et de recherche français ou étrangers, des laboratoires publics ou privés. 


\section{The human NANOS3 germline gene contributes to lung tumour invasion by inducing epithelial-mesenchymal transition}

Simon Grelet ${ }^{1}$, Vanessa Andries ${ }^{2,3}$, Myriam Polette ${ }^{1,4}$, Christine Gilles ${ }^{5}$, Katrien Staes ${ }^{2,3}$, Anne-Pascaline Martin ${ }^{1}$, Claire Kileztky ${ }^{1}$, Christine Terryn ${ }^{6}$, Véronique Dalstein ${ }^{1,4}$, Chun-

Wen Cheng ${ }^{7}$, Chen-Yang Shen ${ }^{7}$, Philippe Birembaut ${ }^{1,4}$, Frans Van Roy ${ }^{2,3}$ and Béatrice Nawrocki-Raby ${ }^{1}$

${ }^{1}$ INSERM UMR-S 903, SFR CAP-Santé, University of Reims Champagne-Ardenne, Reims, F-51100 France

${ }^{2}$ Department of Biomedical Molecular Biology, Ghent University, Ghent, B-9052 Belgium ${ }^{3}$ Molecular Cell Biology Unit, Inflammation Research Center, VIB, Ghent, B-9052 Belgium

${ }^{4}$ Laboratory of Histology, CHU of Reims, Reims, F-51100 France

${ }^{5}$ Laboratory of Developmental and Tumor Biology, GIGA-Cancer, University of Liège, CHU of Sart-Tilman, Liège, B-4000 Belgium

${ }^{6}$ Plateforme Imagerie Cellulaire et Tissulaire, University of Reims Champagne-Ardenne, Reims, F-51100 France

${ }^{7}$ Institute of Biomedical Sciences, Academia Sinica, Taipei 11529, Taiwan

Running title: Nanos3 induces EMT-associated lung tumour invasion

Corresponding author: Béatrice Nawrocki-Raby, INSERM UMR-S 903, CHU Maison Blanche, 45 rue Cognacq-Jay, F-51100 REIMS, France. Phone: +33.3.26.78.77.70; Fax: +33.3.26.06.58.61; e-mail: beatrice.raby@univ-reims.fr

Conflict of interest: The authors declare no conflict of interest. 


\begin{abstract}
In this study, we explore the role of the human NANOS3 germline gene in lung tumour progression. We report that Nanos3 is overexpressed by invasive lung cancer cells and represents a new prognostic marker for non-small cell lung carcinomas (NSCLC). We also show that Nanos3 gene expression is restricted in testis and brain and is regulated by epigenetic events. Moreover, we demonstrate that Nanos3 is upregulated in cultured cells undergoing epithelial-mesenchymal transition (EMT). Nanos3 overexpression in human NSCLC cell lines enhances their invasiveness by upregulating EMT, whereas its silencing induces mesenchymal-epithelial transition. Nanos3 represses E-cadherin at the transcriptional level and upregulates Vimentin post-transcriptionally. Also, we show that Nanos3 binds mRNAs encoding Vimentin and regulates the length of the 3' poly(A) tail of VIM mRNA Finally, Nanos3 can also protect Vimentin mRNA from microRNA-mediated repression. We thus demonstrate a new role for Nanos3 in the acquisition of invasiveness by human lung tumour cells and propose a new mechanism of post-transcriptional regulation of EMT.
\end{abstract}

Keywords: Nanos3; Lung cancer; Tumour invasion; Epithelial-mesenchymal transition; Posttranscriptional regulation. 


\section{INTRODUCTION}

The NANOS genes comprise a small family of evolutionarily conserved RNA-binding proteins required in diverse organisms for regulation of various developmental processes, including embryonic patterning and germ cell development. The first Nanos family member described was the unique nos gene in Drosophila melanogaster, identified as a maternal effect gene required for abdomen formation. Nos has been extensively studied and is now well known to control the differentiation of the anterior-posterior body axis, primordial germ cell migration, maintenance of germline stem cell self-renewal, and suppression of somatic cell fate during germline development [1-5]. In all species analysed, Nanos homologs are characterized by a highly conserved zinc-finger domain. This domain, which mediates protein-nucleic acid interactions, has an essential role in translational regulation of Nanos targets and is also predicted to be involved in protein-protein interactions [6]. In humans, three homologs of Nanos (Nanos1, -2 and -3) have been characterized and shown to have conserved functions in germline development [7-9]. In a tumour context, it was recently shown that Nanos2 and Nanos3 are meiosis regulators and are dysregulated in testicular carcinoma in situ [10]. Moreover, Janic et al. previously showed that ectopic expression of germline genes such as nos drives malignant brain tumour growth in Drosophila [11]. In addition, upregulation of Nanos 1 and Nanos3 enables the oncogenic growth of pRb-deficient cells suggesting that Nanos has a stimulatory role in cancer cell proliferation [12]. Otherwise, we previously reported that human Nanos1 (hNanos1) is repressed by E-cadherin and is involved in the acquisition of invasive properties by lung tumour cells via regulation of matrix metalloproteinase (MMP)-14 expression [13, 14].

During tumour progression, phenotypic changes occur in epithelial tumour cells, enabling only a few of them to overcome successive barriers and develop overt metastases. This phenotypic conversion, so-called EMT (Epithelial-Mesenchymal Transition), is characterized 
by diminished epithelial characteristics and increased mesenchymal attributes, and contributes to enhancement of invasive properties of epithelial tumour cells [15]. During the EMT process, "mesenchymal" markers (such as Vimentin, $\alpha$-smooth muscle actin, fibroblast specific protein 1 and MMPs) are frequently overexpressed whereas epithelial features (such as E-cadherin and Occludin) are dramatically decreased [16, 17]. These EMT targets genes are regulated by several specific transcriptional factors such as Snail, Slug, ZEB1, ZEB2, Twist and others. These proteins can transcriptionally regulate additional targets both by repressing other "epithelial" genes (such as other components of adherens and tight junctions) but also by inducing "mesenchymal” genes such as Vimentin or MMPs [18, 19].

To determine whether Nanos3 is involved in tumour progression, we analysed the expression pattern of Nanos3 in samples of human non-small cell lung carcinoma (NSCLC) and evaluated the relationship between Nanos3 and EMT process. We provide new data on the role of Nanos3 in human lung tumour progression and report evidence for a novel posttranscriptional regulation of Vimentin by Nanos3.

\section{MATERIALS AND METHODS}

\section{Tumour tissue samples}

The frozen tumour pieces used for total protein extraction and subsequent western blot analyses were obtained from samples of the Tumour Bank of the Reims University Hospital Biological Resource Collection No. DC-2008-374. Surgically resected tumours were collected after obtaining informed consent from 24 patients with primary lung adenocarcinoma and 26 patients with squamous-cell carcinoma (SCC). In parallel, nontumoural parenchyma and bronchi samples matching the tumour samples were also collected. This study was approved by the Institutional Review Board of the Reims University Hospital. For 
immunohistochemistry analysis, tissue array slides containing 94 NSCC and 9 matched metastases were purchased from SuperBioChips (SuperBioChips Laboratories, Seoul, Korea).

\section{Cell culture}

Human lung cell lines HBE4-E6/E7, A549, BEAS-2B, NCI-H1299, Calu-1 and SK-LU-1 were obtained from the American Type Culture Collection (Rockville, MD, USA). A description of culture reagents is provided in Supplementary materials and methods. For mRNA stability experiments, Calu-1 and SK-LU-1 were treated with actinomycin D, at 2.5 $\mu \mathrm{g} / \mathrm{ml}$ and $10 \mu \mathrm{g} / \mathrm{ml}$, respectively (Sigma-Aldrich, St. Louis, MS, USA).

\section{Stable transfection of Nanos3 cDNA vector}

The eukaryotic expression plasmid pDEST-NANOS3 was constructed by transferring the human NANOS3 cDNA to the pDEST12.2 vector by Gateway technology (Life Technologies, Carlsbad, CA, USA). Calu-1 and SK-LU-1 cells were stably transfected with pDEST or pDEST-NANOS3 vectors by electroporation and selected with G418 (50 $\mu \mathrm{g} / \mathrm{ml})$.

\section{Transient transfection of Nanos3 cDNA}

Transient transfection of pDEST or pDEST-NANOS3 in A549 cells was carried out with XtremeGENE transfection reagent (Roche Diagnostics, Mannheim, Germany) according to the manufacturer's instructions.

\section{Transfection of siRNAs}

siRNA specific sequences described in Supplementary Table S3 were selected in the coding sequence of NANOS3 (GeneBank accession number: NM_001098622.2) to generate 21nucleotide sense and 21-nucleotide antisense strands of the type (19N)TT (N, gene-specific 
nucleotide) (Eurogentec, Seraing, Belgium). Corresponding scrambled duplexes that do not recognize any sequence in the human genome were used as controls. Cells were transfected with siRNA duplexes $(20 \mathrm{nM})$ by the calcium phosphate precipitation method as previously described [20].

\section{Western blotting}

Total proteins from cells or tumour samples were extracted in RIPA buffer containing complete protease inhibitor cocktail (Roche Diagnostics). The subsequent steps were performed as previously described [20].

\section{Immunohistochemistry}

Immunohistochemistry for Nanos3 was done on paraffin tissue array sections. After antigen retrieval in citrate buffer (Dako, Glostrup, Denmark), subsequent steps were performed with the biotin free EnVision + Dual Link System-HRP kit (Dako), and HRP activity was revealed with AEC+ substrate-chromogen (Dako). A detailed description of semi-quantitative scorings is provided in Supplementary materials and methods. Immunohistochemistry for Vimentin (Dako) and E-cadherin (Dako) was performed using a Ventana Benchmark XT autostainer (Ventana Medical Systems, Inc., Tucson, AZ). Subsequent steps were performed with the UltraView universal DAB detection kit (Ventana).

\section{Real-time qRT-PCR for in vitro analysis}

Total RNA was isolated using the High Pure RNA isolation kit (Roche Diagnostics). Total RNA $(1 \mu \mathrm{g})$ was reverse-transcribed into cDNA with the Transcriptor First Strand cDNA Synthesis kit (Roche Diagnostics). qPCR reactions were performed using the "Fast Start Universal Probe Master" kit and the UPL-probe system as recommended by the manufacturer, 
in a LightCycler 480 Instrument (Roche Diagnostics). Forward and reverse primers (Eurogentec) for NANOS3, CDH1, VIM, GAPDH and 18S are described in Supplementary Table S3. Each sample was quantified using a standard curve based on serial dilutions of a batch of human placenta cDNA.

\section{Epigenetic regulation analysis}

Cell cultures were treated with $1 \mu \mathrm{M}$ 5-aza-2'-deoxycytidine (DAC) (Sigma-Aldrich), $0.1 \mu \mathrm{M}$ Trichostatin A (TSA) (Sigma-Aldrich), $100 \mu \mathrm{M}$ CTK7A (Millipore) or $80 \mu \mathrm{M}$ of Sadenosylmethionine (SAM) (New England Biolabs, Ipswich, MA, USA). DAC, TSA and CTKA7 stock solutions were diluted in DMSO. The SAM solution was prepared in a filtered solution of $5 \mathrm{mM} \mathrm{H} \mathrm{SO}_{4}$ and $10 \%$ ethanol. Cells were treated for four days before RNA extraction and RT-PCR analysis.

\section{Cell circularity analysis}

Cellular circularity was measured with ImageJ software (http://imagej.nih.gov/ij/index.html) according to the following formula: circularity $=4 \pi\left(\right.$ area/perimeter $\left.^{2}\right)$.

\section{Invasion assay}

The in vitro invasive properties of cells were assessed using a Matrigel modified Boyden chamber assay as previously described [21].

\section{D migration assay}

The 2D migration assay has been described [21]. 


\section{EMT-inducible model}

For the 2D migration model of EMT, HBE4-E6/E7 cells were seeded inside a restriction glass ring on coverslip as described above and then treated for 3 days with $20 \mathrm{ng} / \mathrm{ml}$ EGF after removal of the ring. This was followed by methanol fixation and immunofluorescence analysis.

\section{Bioinformatic prediction of Nanos3 localization}

We used the following programs to predict the subcellular localization of Nanos3: ESLPred (http://www.imtech.res.in/raghava/eslpred) [22]; PSORT II (http://psort.nibb.ac.jp) [23]; SubLoc v1.0 (http://www.bioinfo.tsinghua.edu.cn/SubLoc) [24]; CELLO (http://cello.life.nctu.edu.tw/) [25]; Euk-mPLoc (http://www.csbio.sjtu.edu.cn/bioinf/eukmulti-2) [26]; YLoc+ (http://abi.inf.uni-tuebingen.de/Services/YLoc/webloc.cgi) [27].

\section{Subcellular fractionation}

Purified proteins from four subcellular fractions (organelle, cytosolic, cytoskeletal and nuclear fractions) of BEAS-2B cells were obtained by using the ProteoExtract subcellular proteome extraction kit (Merck, Darmstadt, Germany) according to the manufacturer's instructions.

\section{Immunofluorescence and colocalization analysis}

Subconfluent monolayers of cells cultured on glass coverslips were fixed with methanol or 4\% paraformaldehyde for simple labeling or colocalization analysis, respectively, as previously described [21]. For colocalization studies, the staining was analysed using a Zeiss LSM 710 confocal laser scanning microscope with a $63 \mathrm{x}$ oil-immersion objective and the Zeiss operating system associated with the ZEN software program (Carl Zeiss MicroImaging $\mathrm{GmbH}$, Germany). Fifty planes $(0.2 \mu \mathrm{m}$ between each plane) were combined in a 
reconstruction by using the Imaris software for surface reconstruction (Bitplane AG, Zurich, Switzerland).

\section{RNA immunoprecipitation}

Cells were lysed and immunoprecipitations were performed using either control IgG or antiNanos3 antibody according to the instructions for the EZ-Magna RIP RNA-Binding Protein Immunoprecipitation Kit (Millipore, Darmstadt, Germany). The purification of protein complexes was checked by western blot. After phenol/chloroform treatment, RNA extracted from the complexes was subjected to RT-PCR by using the First Strand cDNA Synthesis Kit for RT-PCR (Roche Diagnostics). PCR reactions were performed with the AmpliTaq Gold PCR Master Mix kit (Applied Biosystem). Forward and reverse primers (Eurogentec) for CDH1 and VIM are described in Supplementary Table S3.

\section{mRNA poly(A) tail length analysis}

The lengths of mRNA poly(A) tails were measured using the poly(A) tail length assay kit (Affymetrix Santa Clara, CA, USA). Briefly, $1 \mu \mathrm{g}$ of total RNA was G-I tailed and reverse transcribed using a tail-specific primer. Samples were amplified by PCR using the VIM specific forward primer described in Supplementary Table S3 and the universal reverse primer provided with the kit. An ACTIN specific forward primer provided with the kit was used as control. PCR products were analysed on a 10\% acrylamide gel and visualized on LAS-1000 fluorescence imager (Fujifilm, Tokyo, Japan) after SYBR Gold Nucleic Acid Gel Staining (Invitrogen). 


\section{Dual luciferase reporter assay}

WT and mutated VIM3'-UTR firefly luciferase reporter vectors have been reported [28]. Both Calu-1 and SK-LU-1 transfectants were transfected with 100 ng reporter construct containing WT or mutated 3'-UTR of VIM. The firefly luciferase/Renilla luciferase activity ratio of each sample was measured in the dual-luciferase reporter assay system (Promega, WI, USA).

\section{Statistical analysis}

For in vivo experiments, Mann-Whitney and Wilcoxon tests were used to compare Nanos3 levels by western blot and immunohistochemistry, respectively. Associations between Nanos 3 score and biological data were studied using Chi-square test. Univariate analysis was performed to identify factors associated with overall survival using the Kaplan-Meier method and compared with the Log Rank test. Factors with a $p$-value $<0.05$ by univariate analysis were included in a multivariate analysis using the Cox proportional hazard model. For in vitro experiments, data are expressed as a mean \pm standard deviation, and the one-sample $t$-test or Student's $t$-test was used for statistical analysis. $P<0.05$ was considered significant. Analyses were performed using GraphPad Prism version 5.0 (GraphPad Software, La Jolla, CA) and Stata/IC 10 (StataCorp, College Station, TX).

\section{RESULTS}

\section{Nanos3 is overexpressed by invasive NSCLC cells.}

We analysed Nanos3 expression in a series of human NSCLC samples using frozen material and tissue array slides. Western blot analysis of protein extracts showed that Nanos3 is overexpressed in both human lung adenocarcinoma (median of Nanos3/GAPDH ratio: 0.09, range: $0.00-0.64)$ and squamous cell carcinoma (SCC) (median: 0.11, range: 0.00-0.92), whereas in the non-tumoural counterparts it was almost undetectable: parenchyma (median: 
0.01, range: $0.00-0.06 ; \mathrm{p}<0.001$ ) and bronchi (median: 0.01, range: $0.00-0.10 ; \mathrm{p}<0.01$ ) (Fig. 1A). This overexpression in tumours was also observed at the transcript level by RT-PCR (data not shown). Immunohistochemical analysis revealed that expression of Nanos3 is a frequent event in NSCLC because only 5 cases out of $94(5.3 \%)$ displayed no staining (Supplementary Table S1). Nanos3 was expressed in the nucleus and/or cytoplasm of tumour cells independently of the histological subtype (Fig. 1B). A stronger staining was frequently observed at the invading front of tumour clusters, especially in SCC (Fig. 1B, f, arrowheads). Moreover, establishing a scale of Nanos3 immunostaining intensity showed that Nanos 3 is expressed less in primary lung tumours than in the metastases associated with them (Fig. 1C). The median scores were, respectively, 8 (range: $4-12$ ) and 14 (range: $5-24 ; \mathrm{p}<0.01$ ). The Nanos3 score was not related to the histological type, differentiation status, tumour size, $\mathrm{N}$ status, or tumour node metastasis (TNM) stage of the primary tumours, but, interestingly, it correlated with patient outcome (Fig. 1D and Table 1). A high Nanos3 score was associated with poor survival of patients with NSCLC. Median survival time was 85.5 months (range: 2-149) for patients with a score $<10$, and 38.5 months (range: 4-132) for those with a score $\geq 10(\mathrm{p}=0.0394)$. Moreover, multivariate analysis showed that Nanos3 is an independent prognostic marker for NSCLC ( $\mathrm{p}=0.041$, Table 1).

Further, comparison of NANOS3 gene expression with in vitro invasive capacity of various lung carcinoma cell lines revealed that cells producing the highest NANOS3 mRNA levels were also the most invasive (Fig. 1E).

Overall, these findings indicate an association between Nanos3 overexpression and lung cancer progression, and particularly tumour invasion and dissemination. 


\section{Human Nanos3 expression is restricted to brain and testis in normal adult tissues and is regulated by epigenetic events.}

We also wondered about the Nanos 3 expression profile in normal human tissues. With the exception of testis and brain, NANOS3 transcripts were almost undetectable in healthy adult somatic tissues (Fig. 2A).

Since Nanos3 was found to be ectopically expressed in human NSCLC, we were interested in the mechanisms controlling the level of neo-expression of the NANOS3 gene in lung cancer. For this purpose, several human tumour cell lines were treated with the DNA methyl transferase inhibitor Decitabine (DAC) or the histone deacetylase inhibitor Trichostatin A (TSA), or with both. We found that the mRNA level of NANOS3 was upregulated by DAC in HBE4-E6/E7 cells and by the combination of DAC and TSA in SKLU-1, Calu-1 and A549 cells (Fig. 2B). In contrast, the high level of NANOS3 mRNA expressed by NCI-H1299 cells was unaffected by these inhibitors. Conversely, treatment of NCI-H1299 cells with the methyl donor S-adenosyl methionine (SAM) or the histone acetyl transferase inhibitor VII (CTK7A), or with both, induced a decrease in NANOS3 gene expression (Fig. 2B).

Taken together, these in vitro and in vivo data indicate that NANOS3 gene expression is enriched in testis and brain, and that it is overexpressed in lung cancer by epigenetic mechanisms involving both DNA methylation and histone acetylation.

\section{Nanos3 is upregulated during experimental EMT in vitro and regulates EMT-associated invasion.}

It has been proposed that EMT is very important in tumour invasion [29], so we verified the relationship between Nanos 3 and the EMT process. To this end, we used two in vitro models of EMT in HBE4-E6/E4 bronchial cells: induction of 2D migration (Fig. 3A) 
and epidermal growth factor (EGF) treatment (Supplementary Fig. S1A). In both models, we saw cell morphology changes characteristic of EMT. The loss of membrane-associated expression of the epithelial marker E-cadherin and the de novo expression of the mesenchymal marker Vimentin were used to confirm the occurrence of EMT in both models. Interestingly, we found that Nanos3 was upregulated in cells that underwent EMT (Fig. 3A and Supplementary Fig. S1A).

To study whether Nanos3 is causally involved in tumour cell invasion, we first generated stable transfectants overexpressing Nanos3 in the squamous cell carcinoma cell line Calu-1 and the adenocarcinoma cell line SK-LU-1, which were originally moderately invasive. Nanos3 overexpression induced a drastic morphological change from a cohesive epithelial cell shape to a more scattered fibroblast-like cell shape coupled with decreased cell roundness (Fig. 3B). Western blot analysis revealed downregulation of E-cadherin and overexpression of Vimentin in Nanos3-overexpressing cells (Fig. 3C). Consistent with these findings, cells overexpressing Nanos3 were more invasive in a Matrigel modified Boyden chamber assay (Fig. 3D). Similar results were obtained with A549 cells transiently transfected with NANOS3 cDNA (data not shown).

Conversely, Nanos3 silencing in the highly invasive NCI-H1299 lung tumour cell line by transient transfection of NANOS3 small interfering RNA (siRNA) induced a more epithelioid phenotype characterized by a larger cell circularity index (Fig. 3E). Cells with transiently silenced Nanos3 showed increased expression of E-cadherin as well as decreased expression of Vimentin (Fig. 3F). This was accompanied by a strong decrease in invasive and 2D/3D migratory capacities of Nanos3-silenced cells (Fig. 3G; Supplementary Fig. S1B and S1C; Supplementary Video S1). Similar results were also obtained with BEAS-2B (Supplementary Fig. S2) and A549 (data not shown) cell lines transiently transfected with Nanos3 siRNA. 
In addition, our immunohistochemical analysis of serial sections of human NSCLC showed that all tumor clusters expressing Vimentin and showing loss of E-cadherin also express Nanos3 (Fig. 3H).

\section{Differential regulation of EMT targets by Nanos3.}

To explore the mechanisms of EMT target regulation by Nanos3, we first analysed the mRNA levels of $C D H 1$ and VIM. Contrary to VIM transcripts, $C D H 1$ mRNA levels were dramatically decreased upon Nanos3 overexpression in Calu-1 and SK-LU-1 cells, which indicates that Nanos3 may employ different mechanisms to regulate its targets (Fig. 4A). This hypothesis was supported by RNA immunoprecipitation (RIP) experiments. In BEAS-2B and A549 cells, VIM mRNA co-immunoprecipitated with the Nanos3 protein, whereas CDH1 mRNA did not (Fig. 4B, top). These RIP results were also validated in Nanos3overexpressing Calu-1 cells (Fig. 4B, middle) and in Nanos3-silenced NCI-H1299 and A549 cells (Fig. 4B, bottom).

We also assessed the precise subcellular localization of Nanos 3 by using a combination of bioinformatic prediction methods, subcellular protein fractionation and fluorescence microscopy. In silico, the NNCN, CELLO, Euk-mPLoc, SubLoc, ESLPred, PSORT II, and Yloc+ algorithms [22-27] all predicted that Nanos3 is preferentially localized in the nucleus (Supplementary Table S2). By subcellular fractionation followed by western blotting, we confirmed in the BEAS-2B cell line both nuclear and cytoplasmic localization of Nanos3 (Fig. 4C). Immunofluorescence labeling of Nanos3 in BEAS-2B cells demonstrated moderate cytoplasmic and strong nuclear localization of Nanos3 (Fig. 4D, top). By confocal laser scanning microscopy, the nuclear Nanos3 signal appeared mainly as speckles colocalized with the nucleolar marker Nucleolin (Fig. 4D, middle and bottom). The strong colocalization of these two proteins was further validated by determining Manders' 
$(\mathrm{M} 1=0.998 ; \mathrm{M} 2=0.819)$ and Pearson's coefficients $(\mathrm{r}=0.998)$. These data demonstrate a dual subcellular localization of Nanos3, a finding correlating with the differential mechanisms of EMT target regulation described above.

Finally, we scrutinized the mechanism by which tumour-associated Nanos3 induction modulates VIM mRNA post-transcriptionally. Nanos family members are known to regulate translation by controlling poly(A) tail length [30]. So, we first examined the effect of modulating Nanos3 expression on the length of the VIM mRNA poly(A) tail. We found that the length of the VIM mRNA poly(A) tail was increased by Nanos3 overexpression (Calu-1: $64 \pm 28 \%, \mathrm{p}<0.05 ;$ SK-LU-1: $109 \pm 35 \%, \mathrm{p}<0.05)$ and decreased by its silencing (A549: 31 \pm 14\%, p < 0.05; Calu-1: $27 \pm 4 \%, \mathrm{p}<0.01$; SK-LU-1: $21 \pm 4 \%, \mathrm{p}<0.05)$ (Fig. 5A). We observed that VIM mRNAs were less sensitive to the actinomycin D treatment in Nanos3 transfectants than in their respective control, suggesting that Nanos3 stabilized mRNAs encoding Vimentin (a increase of $123 \pm 23 \%$ and $97 \pm 51 \%$ for Calu-1 and SK-LU-1, respectively; $\mathrm{p}<0.05$ ) (Fig. 5B). We also showed that the induction of Vimentin protein upon Nanos3 overexpression was abolished by cordycepin, a polyadenylation inhibitor (Fig. 5C). Finally, because miR-30a has been shown to downregulate Vimentin expression [28], we explored the link between Nanos3 and miR-30a. Using a luciferase assay, we found that binding of Nanos3 to the 3 '-UTR of the VIM transcript resulted in a strong induction of luciferase activity in Calu-1 and SK-LU-1 cells. Moreover, we observed that the Nanos3

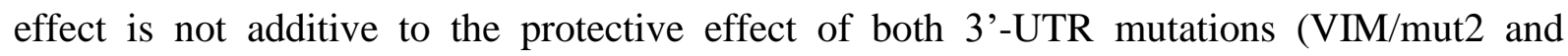
VIM/mut12), reported to affect miR-30a binding sites (Fig. 5D).

All together, these data suggest that Nanos3 regulates Vimentin by a polyadenylationassociated mechanism and protects VIM mRNA from microRNA repression. 


\section{DISCUSSION}

This study demonstrates the involvement of human Nanos3 in the progression of NSCLC. We showed that Nanos3 expression is correlated with tumour invasion in vitro and in vivo and is associated with decreased overall survival of patients. Moreover, Nanos3 is upregulated in vitro during experimentally induced EMT and regulates cell invasion and migration of lung tumour cells via both transcriptional and post-transcriptional regulation of EMT-associated genes.

First, we showed the ectopic expression of the germline gene NANOS3 in NSCLC and its association with a poor outcome. Indeed, ectopic high level expression of Nanos3 is an independent marker of overall survival and may therefore be helpful in prognosis of lung cancer. There is mounting evidence for the ectopic activation of germline genes during progression of several human cancer types [31]. Thus, our study supports this concept and the close similarity between developmental EMT and metastasis-associated EMT. We also showed that Nanos3 reactivation in lung cancer cells is epigenetically regulated by DNA demethylation and histone acetylation. These data are consistent with the fact that tissuespecific patterns of $\mathrm{CpG}$ methylation, which are established during embryonic development, are well preserved in adult somatic cells, but become profoundly altered in cancer cells [32]. For instance, DNA hypomethylation in tumours has been associated with transcriptional activation of several genes, most of which are expressed only in the germline. This group of genes was named cancer-germline (CG) genes and is best known in the lung tumour context $[33,34]$. Interestingly, Nanos3 is strongly expressed by adult testis germ cells but is not expressed in most healthy adult somatic tissues, with the exception of the brain. These findings are in agreement with the previous study of Julaton et al. [9]. Taken together, our data suggest that Nanos3 may be considered a CG gene. 
This study also demonstrates and depicts in part the differential mechanisms by which Nanos3 regulates EMT-associated genes. Indeed, we observed that, in accordance with its dual localization in both nucleus and cytoplasm, Nanos3 transcriptionally downregulates CDH1, but regulates Vimentin post-transcriptionally. Post-transcriptional regulation of EMT is a topic of growing interest, and several mechanisms have emerged as critical in the regulation of EMT during tumour progression [35-37]. It is now well known that the EMT program can be strongly influenced by post-transcriptional mechanisms governed by microRNAs and RNA-binding proteins (RBPs) [36]. The NANOS gene family encodes a group of RBPs believed to function as translational repressors, at least in model organisms such as Drosophila melanogaster [6]. Indeed, the translational repression of hunchback in the fruitfly involves Nanos and Pumilio [38-41]. Here, we found that Nanos3 binds mRNAs encoding Vimentin showing that Nanos3 act as a RBP regulating the EMT process. RBPs are well known for controlling pre-mRNA splicing, capping, and polyadenylation, as well as mRNA export, turnover, localization, and translation [36]. In classical models, RNA-binding Nanos proteins form multisubunit translation-inhibitory complexes with the RNA-binding protein Pumilio, and control the stability and the translation of target mRNAs through regulation of their poly(A) tail length $[6,30]$. In this study, we show that $V I M$ mRNAs are more stable in the Nanos3 transfectants and that Nanos3 regulates the poly(A) tail length of mRNA encoding Vimentin. Our findings demonstrate that the mechanism of poly(A) tail regulation of mRNAs by Nanos family members, which is conserved across multiple species, might be dysregulated during human tumour progression. While Nanos proteins are rather known to promote mRNA deadenylation in normal cell development [30, 42], we report here the opposite effect, with VIM mRNA poly(A) tail lengthening under the influence of Nanos3. This may be due to the involvement of molecular partners of Nanos3 other than those active in the developmental stages of model organisms. In addition, it was recently reported that 
Nanos targets the CNOT6 deadenylase for degradation, allowing stable retention of inherited transcripts in primordial germ cells [43]. In agreement with the previous study showing that Vimentin is dowregulated by miR-30a [28], we also showed that Nanos3, as a RBP, protects VIM mRNAs from miR-30a repression. As miRNAs have been reported to play an important role in the control of cancer progression [37], our finding that Nanos3 can counteract their action is of particular interest. Thus, our results describe new mechanisms of gene regulation by Nanos3 in the human EMT-associated lung tumour invasion context: a repressive regulatory role at the transcriptional level for E-cadherin and a promoting regulatory role at the post-transcriptional level for Vimentin.

Taken together, our findings indicate that the oncofetal induction of human Nanos3 could be useful as a new prognostic marker for NSCLC. Our study also provides new insights into the post-transcriptional mechanisms of cancer-associated EMT induction.

\section{ACKNOWLEDGEMENTS}

This study was supported by Région Champagne-Ardenne, La Ligue Contre le Cancer (Comités de la Marne, Haute-Marne, Meurthe-et-Moselle et Yonne), Lions Club of Soissons, Villers-Cotterets, Château-Thierry and Crépy en Valois, Un Euro contre le Cancer, Fond National pour la Santé ACI 2004-2010 INCa (Cancéropôle Grand-Est project), Research Foundation - Flanders (FWO-Vlaanderen), Foundation against Cancer - Belgium, Fonds National de la Recherche Scientifique (FRS-FNRS, Belgium) and Partenariat Hubert CurienTournesol. The authors thank Eymeric Lagonotte (INSERM UMRS 903) for technical assistance and Amin Bredan (VIB IRC) for critical reading and editing of the manuscript. 


\section{AUTHOR CONTRIBUTION STATEMENT}

SG, PB, FVR and BNR designed the study. SG, VA, KS, APM, CK, CT, C-W C, C-Y S and BNR performed the experiments. SG, VA, MP, CG, VD, C-W C, C-Y S, PB, FVR and BNR analysed data. All authors were involved in writing the manuscript and had final approval of the submitted version. 


\section{REFERENCES}

1. Lehmann R, Nusslein-Volhard C. The maternal gene nanos has a central role in posterior pattern formation of the Drosophila embryo. Development 1991; 112: 679-691.

2. Gavis ER, Lehmann R. Translational regulation of nanos by RNA localization. Nature 1994; 369: 315-318.

3. Asaoka-Taguchi M, Yamada M, Nakamura A, et al. Maternal Pumilio acts together with Nanos in germline development in Drosophila embryos. Nat Cell Biol 1999; 1: 431-437.

4. Hayashi Y, Hayashi M, Kobayashi S. Nanos suppresses somatic cell fate in Drosophila germ line. Proc Natl Acad Sci U S A 2004; 101: 10338-10342.

5. Wang $Z$, Lin $H$. Nanos maintains germline stem cell self-renewal by preventing differentiation. Science 2004; 303: 2016-2019.

6. Curtis D, Treiber DK, Tao F, et al. A CCHC metal-binding domain in Nanos is essential for translational regulation. EMBO J 1997; 16: 834-843.

7. Jaruzelska J, Kotecki M, Kusz K, et al. Conservation of a Pumilio-Nanos complex from Drosophila germ plasm to human germ cells. Dev Genes Evol 2003; 213: 120-126.

8. Kusz KM, Tomczyk L, Sajek M, et al. The highly conserved NANOS2 protein: testisspecific expression and significance for the human male reproduction. Mol Hum Reprod 2009; 15: 165-171.

9. Julaton VT, Reijo Pera RA. NANOS3 function in human germ cell development. Hum Mol Genet 2011; 20: 2238-2250. 
10. Jorgensen A, Nielsen JE, Almstrup K, et al. Dysregulation of the mitosis-meiosis switch in testicular carcinoma in situ. J Pathol 2013; 229: 588-598.

11. Janic A, Mendizabal L, Llamazares S, et al. Ectopic expression of germline genes drives malignant brain tumor growth in Drosophila. Science 2010; 330: 1824-1827.

12. Miles WO, Korenjak M, Griffiths LM, et al. Post-transcriptional gene expression control by NANOS is up-regulated and functionally important in pRb-deficient cells. EMBO J 2014; 33: $2201-2215$.

13. Strumane K, Bonnomet A, Stove C, et al. E-cadherin regulates human Nanos1, which interacts with p120ctn and induces tumor cell migration and invasion. Cancer Res 2006; 66: 10007-10015.

14. Bonnomet A, Polette M, Strumane K, et al. The E-cadherin-repressed hNanos1 gene induces tumor cell invasion by upregulating MT1-MMP expression. Oncogene 2008; 27: 3692-3699.

15. Kalluri R, Weinberg RA. The basics of epithelial-mesenchymal transition. J Clin Invest 2009; 119: 1420-1428.

16. Gilles C, Newgreen D, Sato H, et al. Matrix metalloproteases and Epithelial-tomesenchymal transition: implications for carcinoma metastasis. In Rise and Fall of Epithelial Phenotype: concepts of Epithelial-Mesenchymal Transition, Savagner P (ed). Eurekah.com and Kluwer Academic/Plenum, 2005; 1-19.

17. Polette M, Mestdagt M, Bindels S, et al. Beta-catenin and ZO-1: shuttle molecules involved in tumor invasion-associated epithelial-mesenchymal transition processes. Cells Tissues Organs 2007; 185: 61-65. 
18. Peinado H, Olmeda D, Cano A. Snail, Zeb and bHLH factors in tumour progression: an alliance against the epithelial phenotype? Nat Rev Cancer 2007; 7: 415-428.

19. Bindels S, Mestdagt M, Vandewalle C, et al. Regulation of vimentin by SIP1 in human epithelial breast tumor cells. Oncogene 2006; 25: 4975-4985.

20. Polette M, Gilles C, Nawrocki-Raby B, et al. Membrane-type 1 matrix metalloproteinase expression is regulated by zonula occludens-1 in human breast cancer cells. Cancer Res 2005; 65: 7691-7698.

21. Joannes A, Bonnomet A, Bindels S, et al. Fhit regulates invasion of lung tumor cells. Oncogene 2010; 29: 1203-1213.

22. Bhasin M, Raghava GP. ESLpred: SVM-based method for subcellular localization of eukaryotic proteins using dipeptide composition and PSI-BLAST. Nucleic Acids Res 2004; 32: W414-W419.

23. Nakai K, Horton P. PSORT: a program for detecting sorting signals in proteins and predicting their subcellular localization. Trends Biochem Sci 1999; 24: 34-36.

24. Chen H, Huang N, Sun Z. SubLoc: a server/client suite for protein subcellular location based on SOAP. Bioinformatics 2006; 22: 376-377.

25. Yu CS, Chen YC, Lu CH, et al. Prediction of protein subcellular localization. Proteins 2006; 64: 643-651.

26. Chou KC, Shen HB. A new method for predicting the subcellular localization of eukaryotic proteins with both single and multiple sites: Euk-mPLoc 2.0. PLoS One 2010; 5: e9931. 
27. Briesemeister S, Rahnenfuhrer J, Kohlbacher O. YLoc--an interpretable web server for predicting subcellular localization. Nucleic Acids Res 2010; 38: W497-W502.

28. Cheng CW, Wang HW, Chang CW, et al. MicroRNA-30a inhibits cell migration and invasion by downregulating vimentin expression and is a potential prognostic marker in breast cancer. Breast Cancer Res Treat 2012; 134: 1081-1093.

29. Nieto MA. Epithelial plasticity: a common theme in embryonic and cancer cells. Science 2013; 342: 1234850 .

30. Wreden C, Verrotti AC, Schisa JA, et al. Nanos and pumilio establish embryonic polarity in Drosophila by promoting posterior deadenylation of hunchback mRNA. Development 1997; 124: 3015-3023.

31. Wang J, Rousseaux S, Khochbin S. Sustaining cancer through addictive ectopic gene activation. Curr Opin Oncol 2014; 26: 73-77.

32. Muntean AG, Hess JL. Epigenetic dysregulation in cancer. Am J Pathol 2009; 175: 13531361.

33. Loriot A, Boon T, De SC. Five new human cancer-germline genes identified among 12 genes expressed in spermatogonia. Int J Cancer 2003; 105: 371-376.

34. Rousseaux S, Debernardi A, Jacquiau B, et al. Ectopic activation of germline and placental genes identifies aggressive metastasis-prone lung cancers. Sci Transl Med 2013; 5: 186 ra66.

35. Evdokimova V, Tognon CE, Sorensen PH. On translational regulation and EMT. Semin Cancer Biol 2012; 22: 437-445. 
36. Aparicio LA, Abella V, Valladares M, et al. Posttranscriptional regulation by RNAbinding proteins during epithelial-to-mesenchymal transition. Cell Mol Life Sci 2013; 70: 4463-4477.

37. De Craene B., Berx G. Regulatory networks defining EMT during cancer initiation and progression. Nat Rev Cancer 2013; 13: 97-110.

38. Tautz D. Regulation of the Drosophila segmentation gene hunchback by two maternal morphogenetic centres. Nature 1988; 332: 281-284.

39. Irish V, Lehmann R, Akam M. The Drosophila posterior-group gene nanos functions by repressing hunchback activity. Nature 1989; 338: 646-648.

40. Barker DD, Wang C, Moore J, et al. Pumilio is essential for function but not for distribution of the Drosophila abdominal determinant Nanos. Genes Dev 1992; 6: 2312-2326.

41. Sonoda J, Wharton RP. Recruitment of Nanos to hunchback mRNA by Pumilio. Genes Dev 1999; 13: 2704-2712.

42. Bhandari D, Raisch T, Weichenrieder O, et al. Structural basis for the Nanos-mediated recruitment of the CCR4-NOT complex and translational repression. Genes Dev 2014; 28: 888-901.

43. Swartz SZ, Reich AM, Oulhen N, et al. Deadenylase depletion protects inherited mRNAs in primordial germ cells. Development 2014; 141: 3134-3142. 
Table 1: Factors associated with overall survival

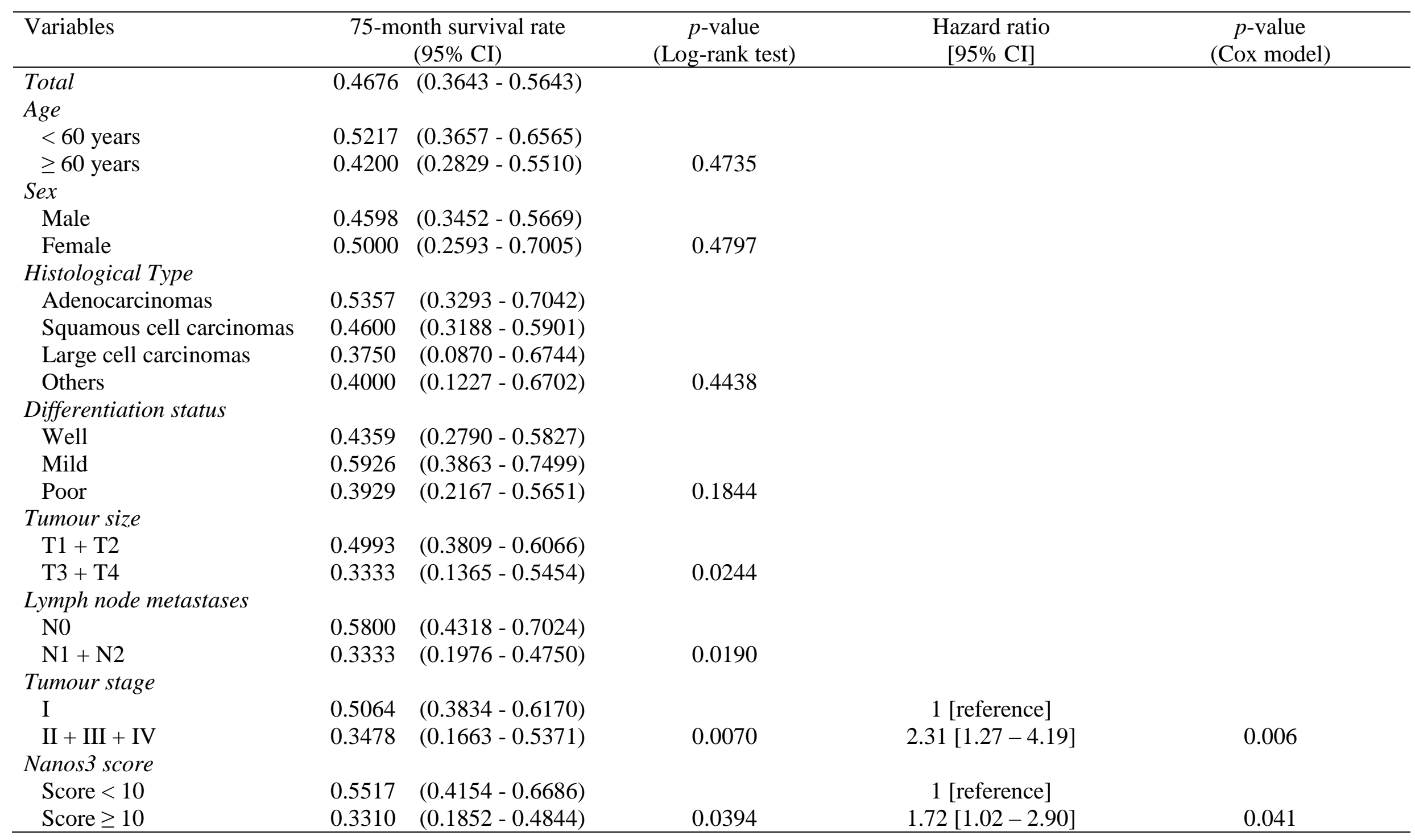




\section{FIGURE LEGENDS}

Figure 1: Nanos3 is overexpressed in non-small cell lung carcinoma tumour cells and is associated with in vivo and in vitro cell invasion.

A, Western blot analysis of Nanos3 levels, normalized versus GAPDH, in protein extracts of a series of adenocarcinomas (ADC) and squamous cell carcinomas (SCC) and of their respective non-tumour counterparts: parenchyma ( $\mathrm{P}$, left panel) and bronchi (B, right panel). Bar, median, $\mathrm{P}: \mathrm{n}=21$; ADC: $\mathrm{n}=24 ; \mathrm{B}: \mathrm{n}=12$, SCC: $\mathrm{n}=26 .{ }^{*} \mathrm{P}<0.01 ; * * * \mathrm{P}<0.001$. B, Immunohistochemical analysis of Nanos3 expression and localization in NSCLC. Human testis tissue (a) served as a positive control and lung parenchyma (b) and bronchus (d) as respective negative controls for adenocarcinomas and squamous cell carcinomas. Nanos3 was expressed in the nucleus and cytoplasm of tumour cells in a well differentiated adenocarcinoma (c) and a moderately differentiated squamous cell carcinoma (e). Nanos3 was mainly expressed at the invading front (arrowheads) of a tumour cluster in a well differentiated squamous cell carcinoma (f). Scale bars, $50 \mu \mathrm{m}$. C, Immunohistochemical comparison of Nanos3 expression in primary tumours (PT) of NSCLC and their matched metastasis (Met). A primary adenocarcinoma (a) and a primary squamous cell carcinoma (c) showed weaker Nanos3 staining than their respective matched bone (b) and lymph node (d) metastases. Scale bars, $50 \mu \mathrm{m}$. For statistical analysis, data were expressed as immunohistochemical (IHC) score (see Materials and Methods). Bars, median, PT: $\mathrm{n}=9$, Met: $\mathrm{n}=9 . * * \mathrm{P}<0.01$. D, Kaplan-Meier survival curve based on immunohistochemical analysis of Nanos3 expression in 94 NSCLC samples with a cut-off at immunostaining score 10 (66.66th percentile: 2/3-1/3 partition of the samples). E, Nanos3 mRNA expression levels in several lung cancer cell lines as analysed by RT-PCR (top), and compared to their invasive capacities tested in a modified Boyden chamber assay (bottom). Error bars, S.D., n = 3 . 
Figure 2: Human Nanos3 expression is restricted to brain and testis in normal adult tissues and is regulated by epigenetic events.

A, qRT-PCR analysis of NANOS3 mRNA level in samples from 20 normal human adult tissues. Gene expression levels were normalized using the geometric mean of the most stable reference genes. CNRQ, calibrated normalized relative quantity. Error bars, S.E.M., n = 3. B, Top, RT-PCR analysis of NANOS3 mRNA levels in HBE4-E6/E7, SK-LU-1, Calu-1, A549 and NCI-H1299 cells treated with the DNA methyl transferase inhibitor Decitabine (DAC), the histone deacetylase inhibitor Trichostatin A (TSA), or with both. Controls were treated with dimethylsulfoxide (DMSO). GAPDH served as internal control. Bottom, NCI-H1299 cells were treated with either the methyl donor S-adenosyl methionine (SAM), the histone acetyl transferase inhibitor VII (CTK7A), or with both. Controls were treated with DMSO + $\mathrm{H}_{2} \mathrm{SO}_{4}+$ ethanol. GAPDH served as internal control. The bar graphs at the right show quantification of NANOS3 mRNA levels normalized to GAPDH for representative experiments.

Figure 3: Nanos3 is overexpressed during EMT induced in vitro and regulates EMTassociated invasion.

A, EMT was induced in the human bronchial cell line HBE4-E6/E7 using a 2D migration model that allows discrimination between stationary and migratory populations of cells. Cells cultured on a glass coverslip were subjected to immunofluorescence detection of Nanos3, Ecadherin and Vimentin. Nuclei were counterstained with DAPI. Scale bars, $50 \mu \mathrm{m}$. B, The circularity index of Calu-1 and SK-LU-1 transfectants overexpressing empty vector (pDEST) or Nanos3 cDNA (NANOS3) was determined from phase contrast photographs using the ImageJ software. Scale bar, $20 \mu \mathrm{m}$; Error bars, S.D., $\mathrm{n}=20$. *** $\mathrm{P}<0.001 . \mathbf{C}$, Western blot 
analysis of Nanos3, E-cadherin, and Vimentin protein levels in Calu-1 and SK-LU-1 transfectants. The levels of GAPDH served as loading control. D, Invasive properties of Calu1 and SK-LU-1 transfectants tested in a Matrigel modified Boyden chamber assay. Data are expressed as fold induction relative to the respective controls. Error bars, S.D., $\mathrm{n}=3$. $* \mathrm{P}<$ 0.05. E, The circularity index of NCI-H1299 transfected with scrambled (scr 1 and scr 2) or Nanos3 siRNA (siRNA 1 and 2) was determined in vitro from phase contrast photographs using the ImageJ software. Scale bar, $20 \mu \mathrm{m}$; Error bars, S.D., $\mathrm{n}=80 . * * * \mathrm{P}<0.001$. F, Western blot analysis of the effect of Nanos3-targeting siRNA 1 and siRNA 2 or scrambled siRNA scr 1 and scr 2 on E-cadherin and Vimentin protein levels in NCI-H1299 cells. The levels of GAPDH served as loading controls. G, Analysis of the effect of inhibiting Nanos3 by siRNA 1 and siRNA 2 on the invasive capacity of NCI-H1299 cells in a modified Boyden chamber invasion assay. Data are expressed as fold induction relative to the respective controls. Error bars, S.D., $\mathrm{n}=4$. $* * \mathrm{P}<0.01$. H, Immunohistochemical analysis of the expression of Nanos3 (a), E-cadherin (b) and Vimentin (c) on serial sections of a NSCLC. Scale bar, $50 \mu \mathrm{m}$.

\section{Figure 4: Differential regulation of EMT targets by Nanos3.}

A, qRT-PCR analysis of CDH1 and VIM mRNA levels in Calu-1 and SK-LU-1 cells transfected with empty vector (pDEST) or Nanos3 cDNA (NANOS3). GAPDH served as an internal control. Error bars, S.E.M., $\mathrm{n}=6$. $* * * \mathrm{P}<0.001$. B, RNA immunoprecipitation (RIP) of Nanos3 protein followed by RT-PCR analysis of the Nanos3-bound mRNAs in coimmunoprecipitation complexes from BEAS-2B and A549 cell lines (top), from Nanos3overexpressing Calu-1 cells (middle), and from NCI-H1299 and A549 cells with Nanos3 knockdown (bottom). C, Distribution of Nanos3 among BEAS-2B subcellular fractions. Cells were fractionated into cytoplasmic (C), membrane/organelle (M), nuclear (N) and cytoskeletal 
matrix (CSK) fractions, and the fractions were analysed by western blotting for Nanos3. Efficiency of the fractionation was controlled using the cytoplasmic marker GAPDH, the membrane marker $\alpha 1-\mathrm{Na} / \mathrm{K}-\mathrm{ATPase}$, the nuclear marker PARP, and the cytoskeletal marker Vimentin. Total cell lysate (T) served as positive control. D, Immunofluorescence analysis of subcellular expression of Nanos3 (red) and/or Nucleolin (green) in BEAS-2B cells by epifluorescence (top) and confocal laser scanning microscopy (middle). Scale bars, $5 \mu \mathrm{m}$. Surface reconstruction of Nanos3/Nucleolin staining with IMARISTM software (bottom). Scale bars, $10 \mu \mathrm{m}$. Nuclei were counterstained with DAPI.

Figure 5: Vimentin is induced by Nanos3 through a lengthening of its mRNA poly(A) tail and a protection from miR-30a-mediated repression.

A, top, Analysis of poly(A) tail length of VIM mRNA (Top) and ACTIN mRNA (Bottom) in siRNA transfectants of A549, control (pDEST) or NANOS3 overexpressing Calu-1, and control (PDEST) or NANOS3 overexpressing SK-LU-1 cells. As indicated on top of the lanes, either scrambled 1 (scr 1) or Nanos3 siRNA 1 (Nanos3 si 1) transfections were performed. Relative intensity of poly(A) tails was measured with ImageJ software. Curves are the means of 3 experiments. Bottom, qRT-PCR analysis of NANOS3 mRNA level in A549, Calu-1 and SK-LU-1 cells transfected as indicated on top. GAPDH served as an internal control. B, Analysis of VIM mRNA stability after treatment of Calu-1 and SK-LU-1 transfectants with actinomycin D. A representative experiment is shown. C, Western blot analysis of the Vimentin protein levels in A549, Calu-1 and SK-LU-1 cells, transfected with empty vector pDEST or with Nanos 3 cDNA, after treatment for $6 \mathrm{~h}$ with the polyadenylation inhibitor cordycepin $\left(100 \mu \mathrm{g} / \mathrm{mL}\right.$, Sigma). Controls were treated with $\mathrm{H}_{2} \mathrm{O}$. The levels of Actin served as loading controls. D, left, Sequences of miR-30a binding sites in the VIM 3'UTR WT and the two mutants VIM 3'UTR/Mut2 and VIM 3'UTR/Mut12 with a mismatch 
at site 2 or at sites 1 and 2, respectively. Right, VIM 3'UTR reporter assay. pDEST and pDEST-NANOS3 transfectants of Calu-1 and SK-LU-1 cells were subjected to a transient transfection with the WT or mutated VIM 3'UTR firefly luciferase reporter vector, followed by a luciferase activity assay. The normalized data are expressed as fold induction relative to the control. Error bars, S.D., $\mathrm{n}=3 . * * \mathrm{P}<0.01 ; * * * \mathrm{P}<0.001$. 
A
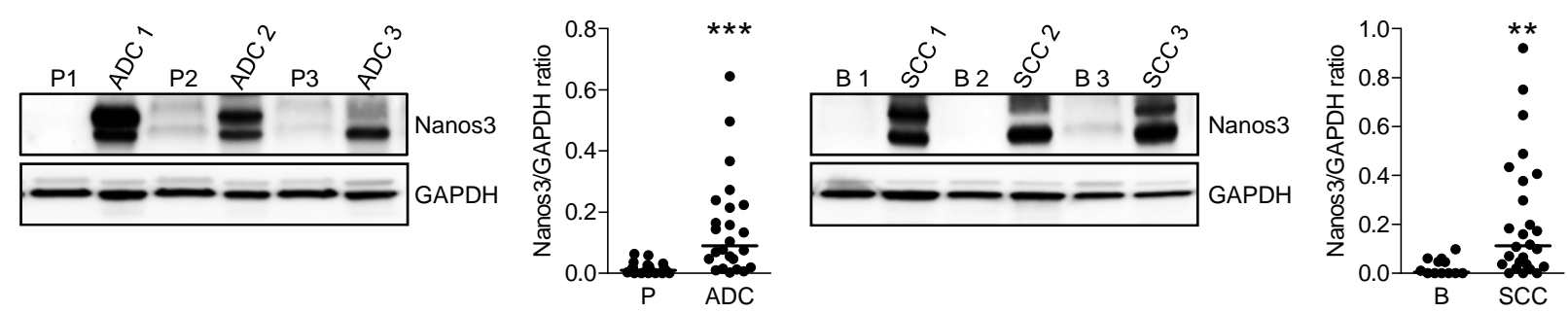

\section{B}
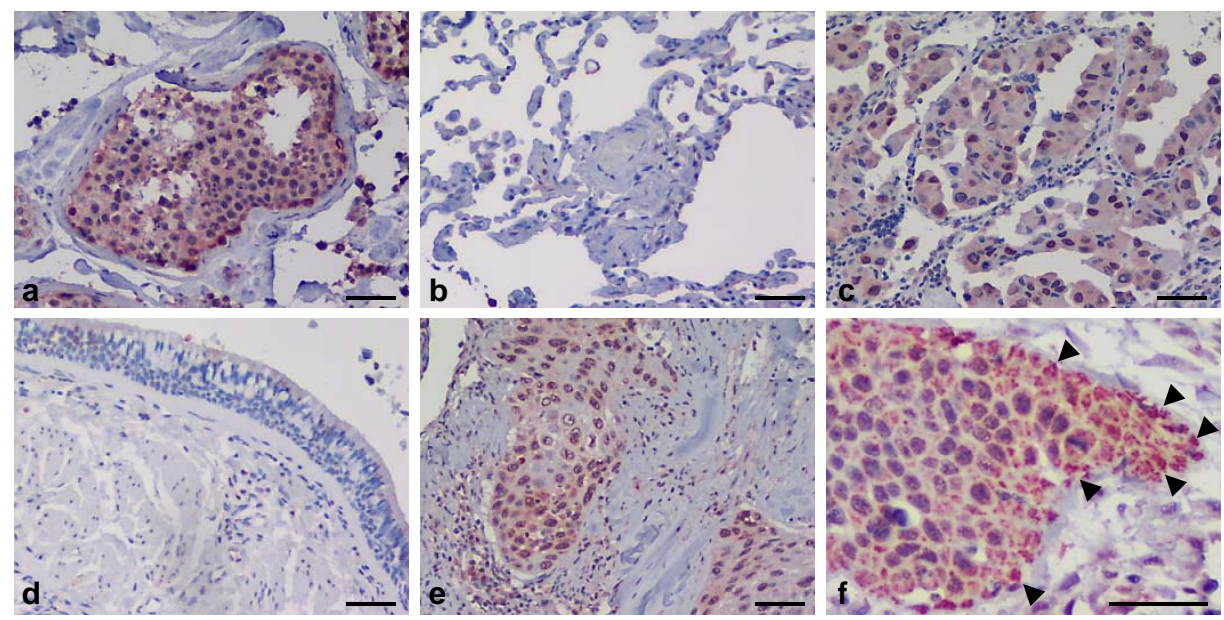

C
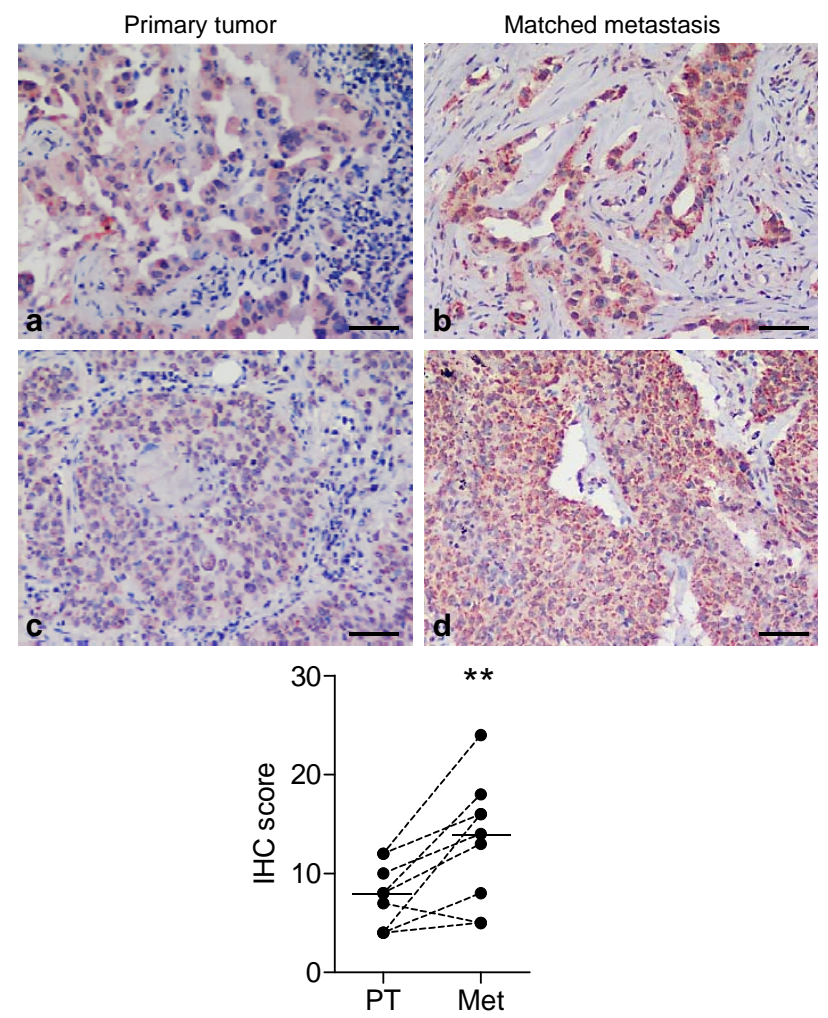

D

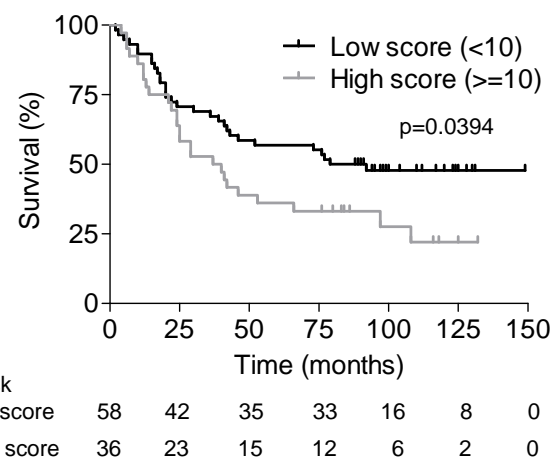

E
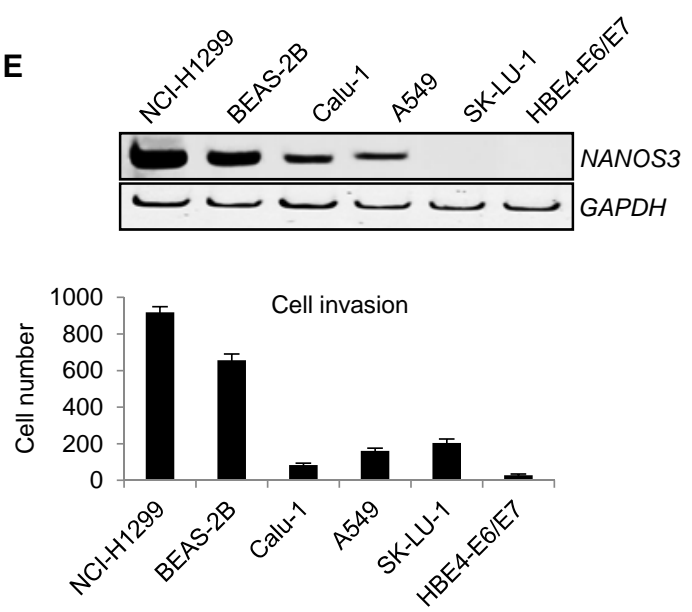

Grelet et al., Figure 1 
A

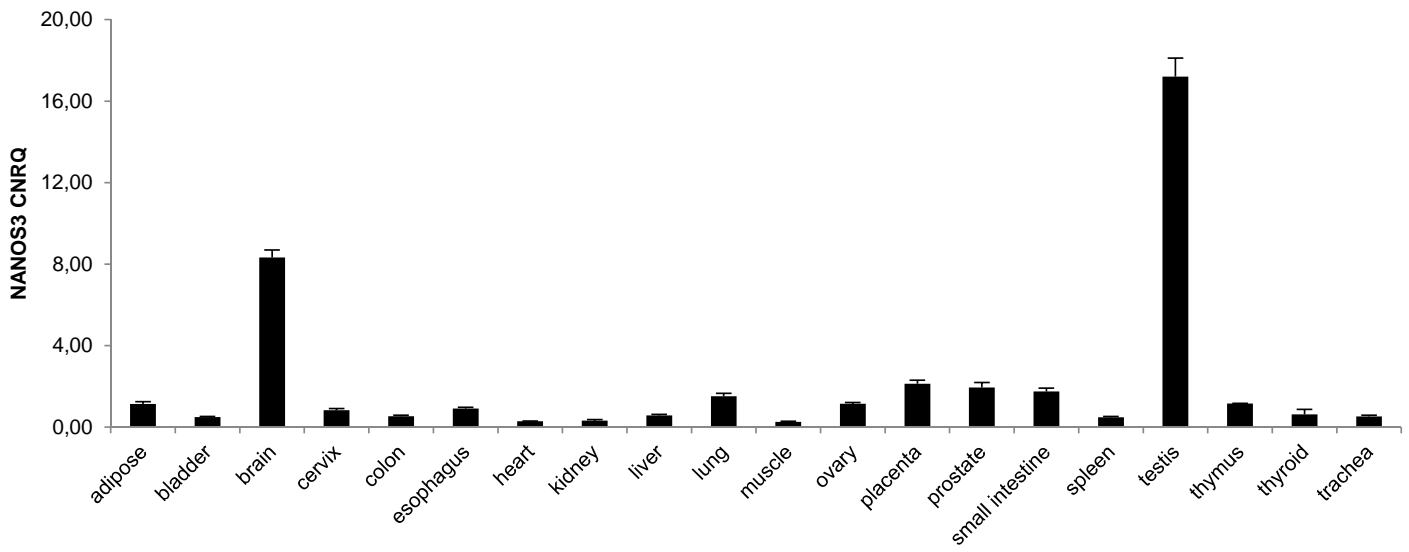

B

Epigenetic reactivation

DNMT \& HDAC inhibitors
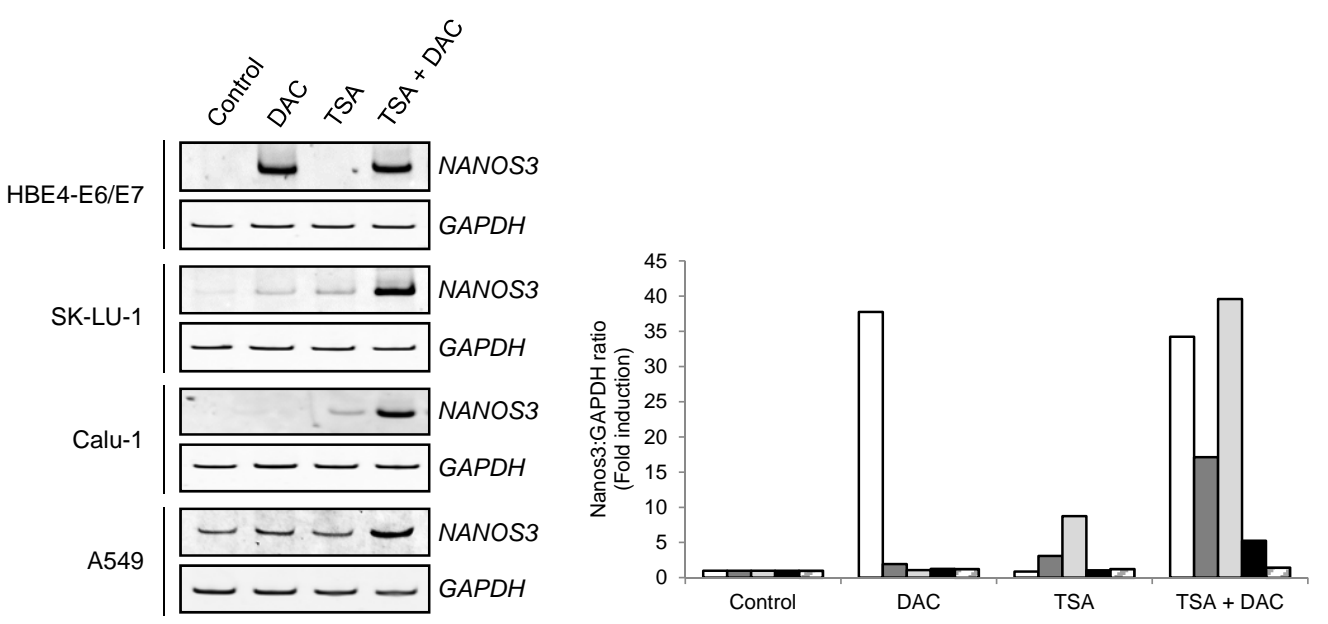

口HBE4-E6/E7

口SK-LU-1

口Calu-1

Calu-1

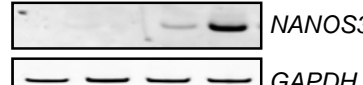

A549

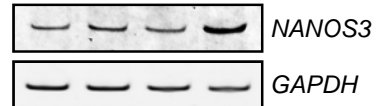

$\mathrm{NCl}-\mathrm{H} 1299$
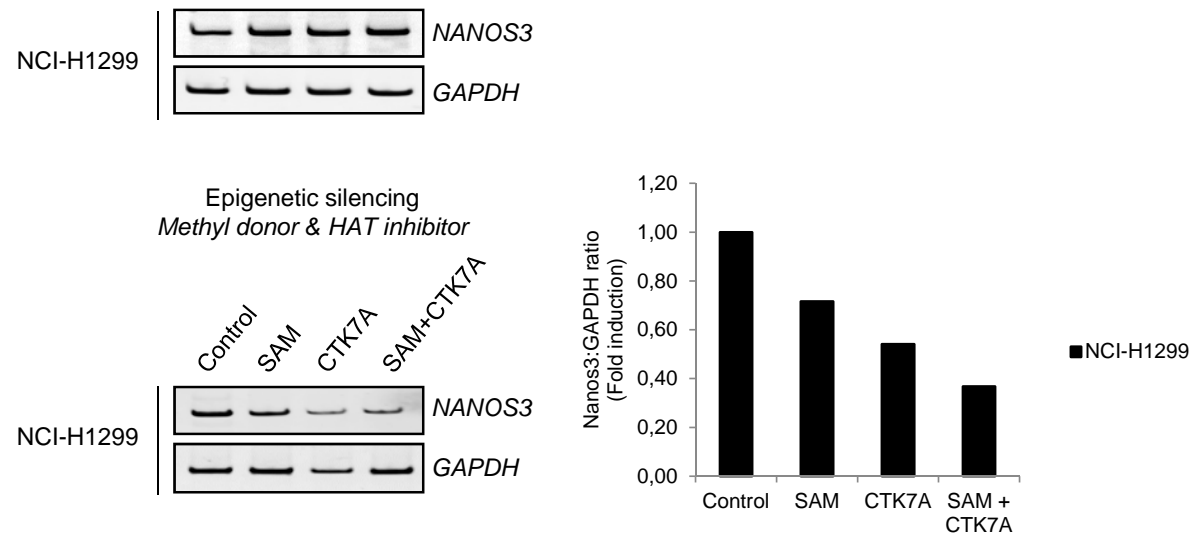
A

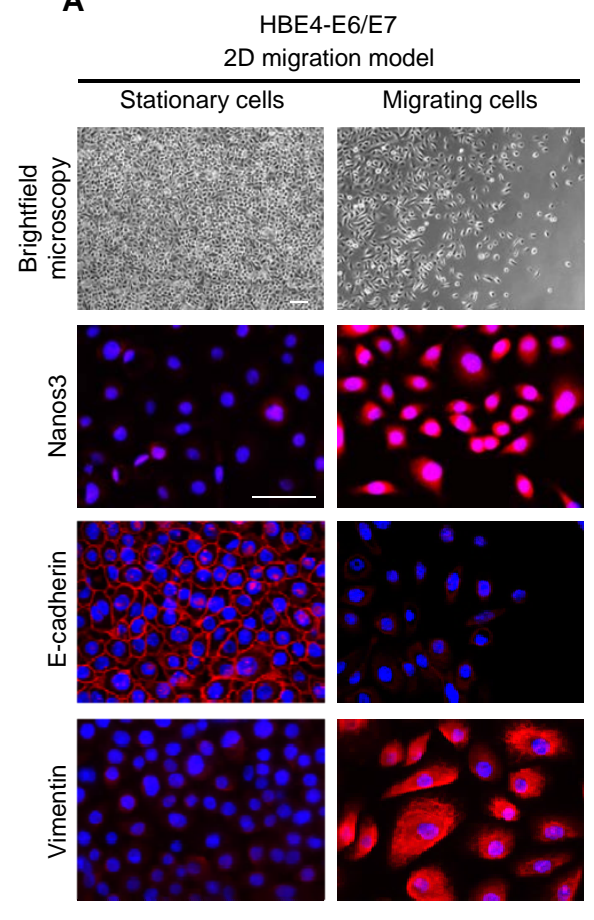

E

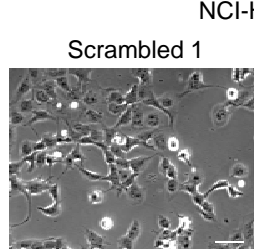

Scrambled 2
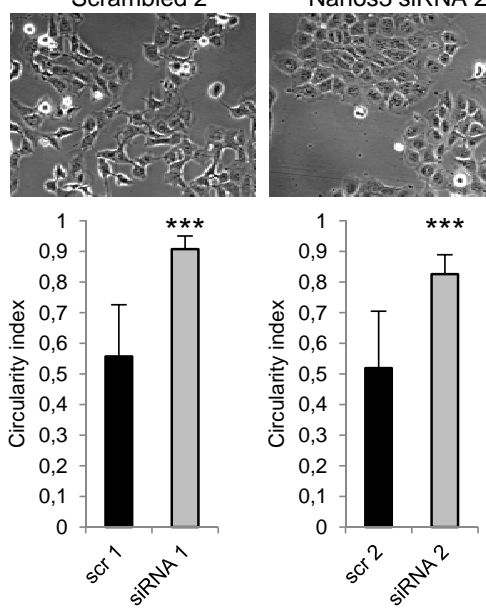

Nanos3 siRNA 2

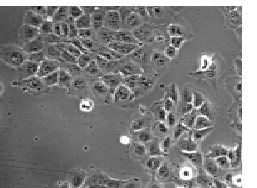

B
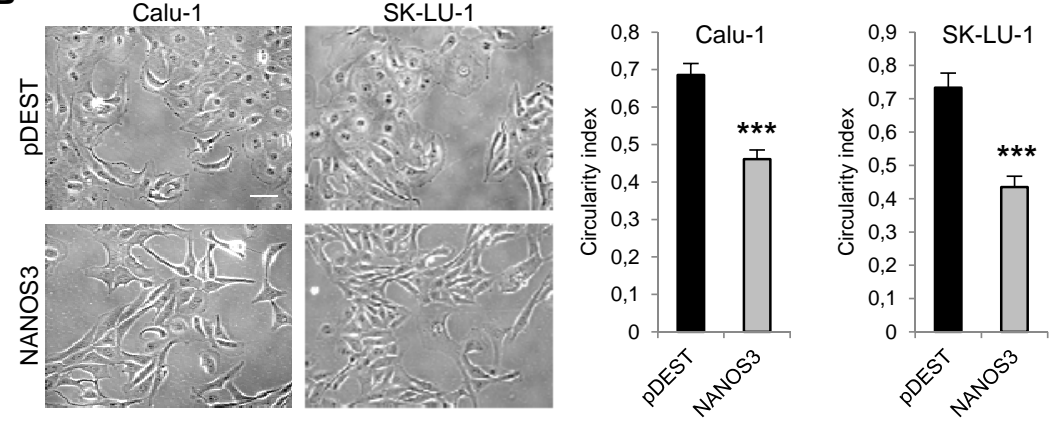

C

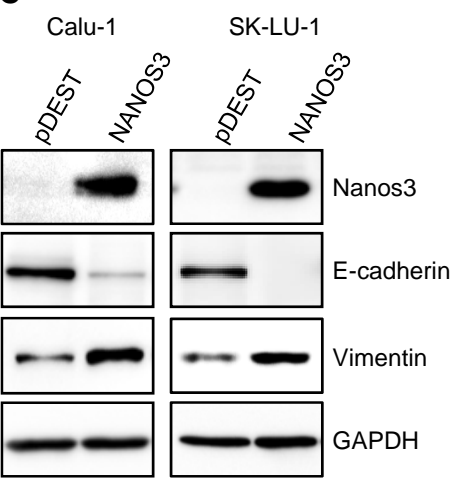

D

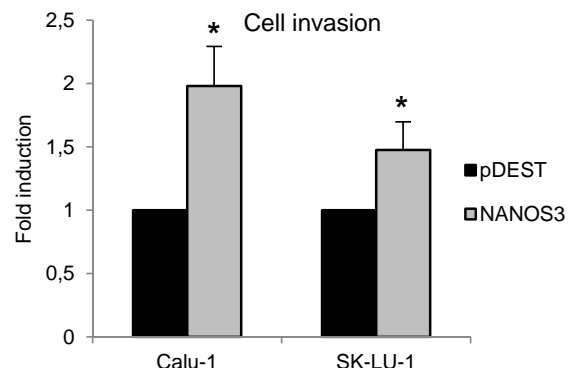

F NCl-H1299

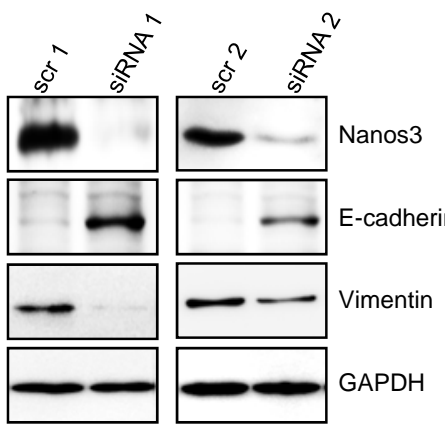

G
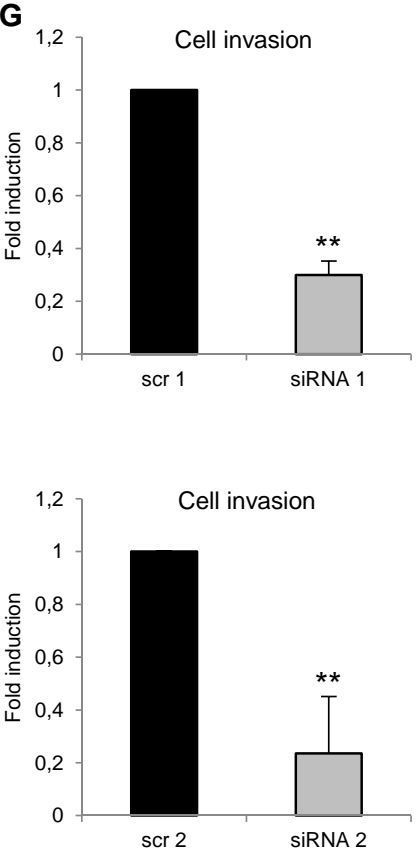

H

Nanos3

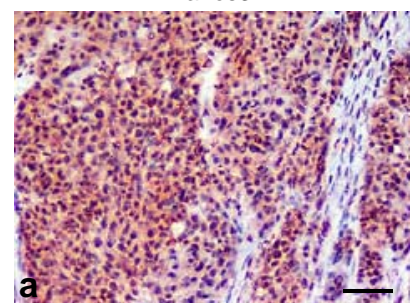

E-cadherin

Vimentin

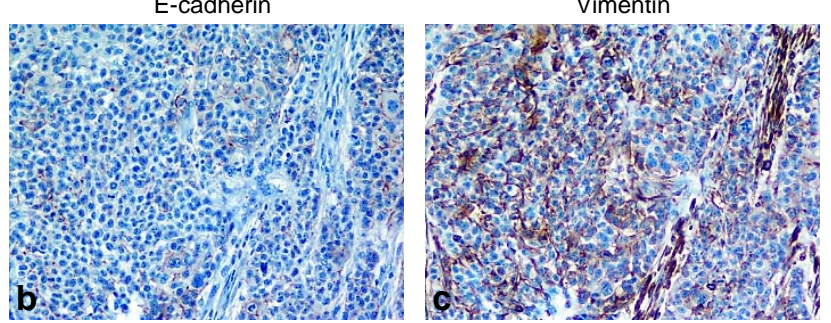



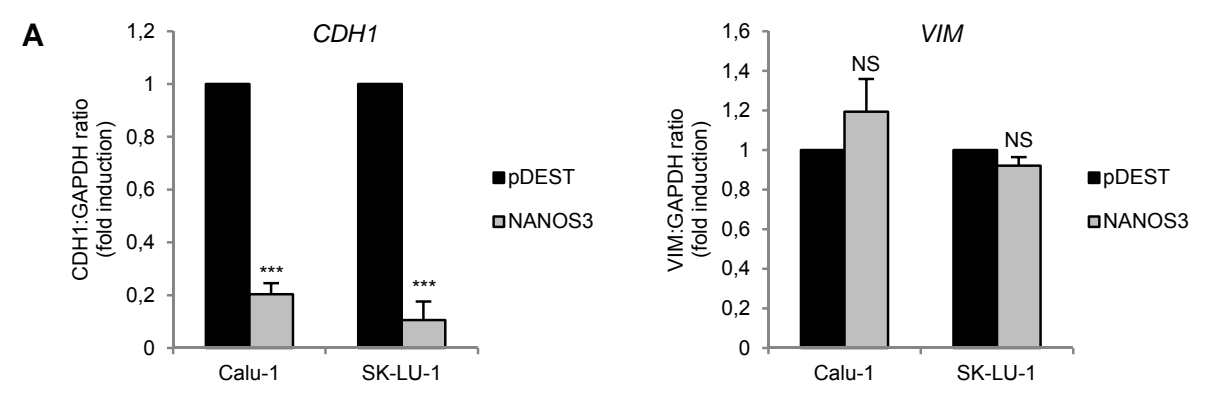

B
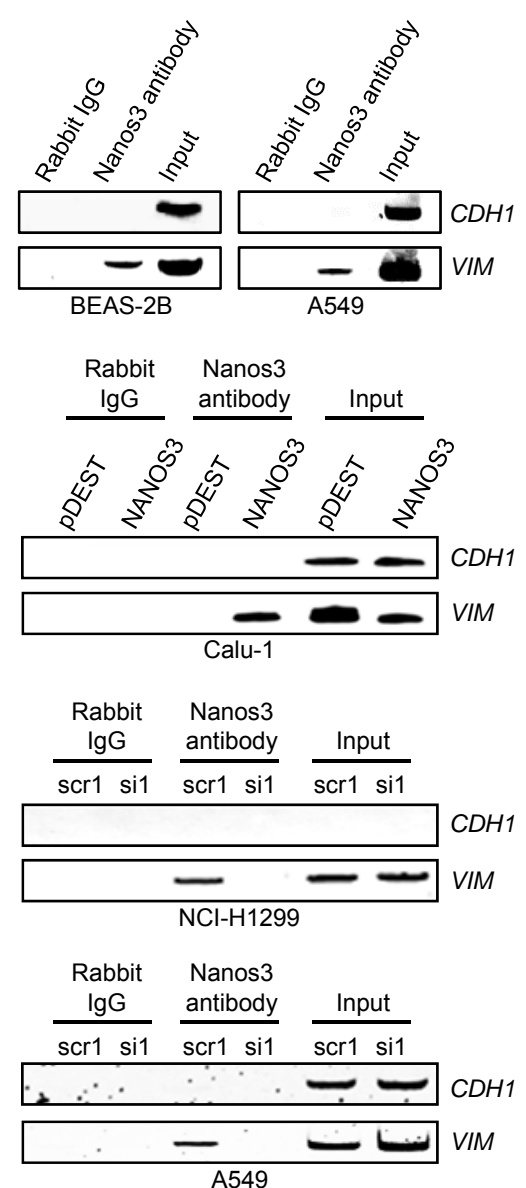

C

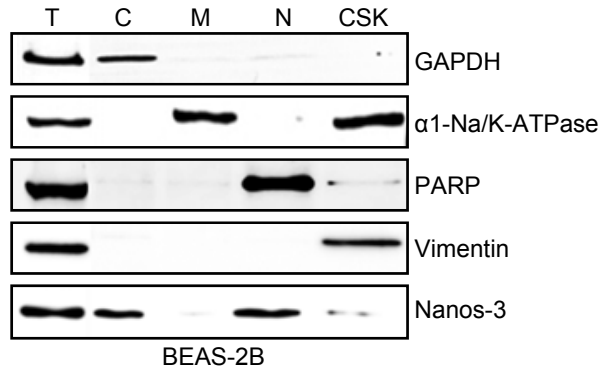

D
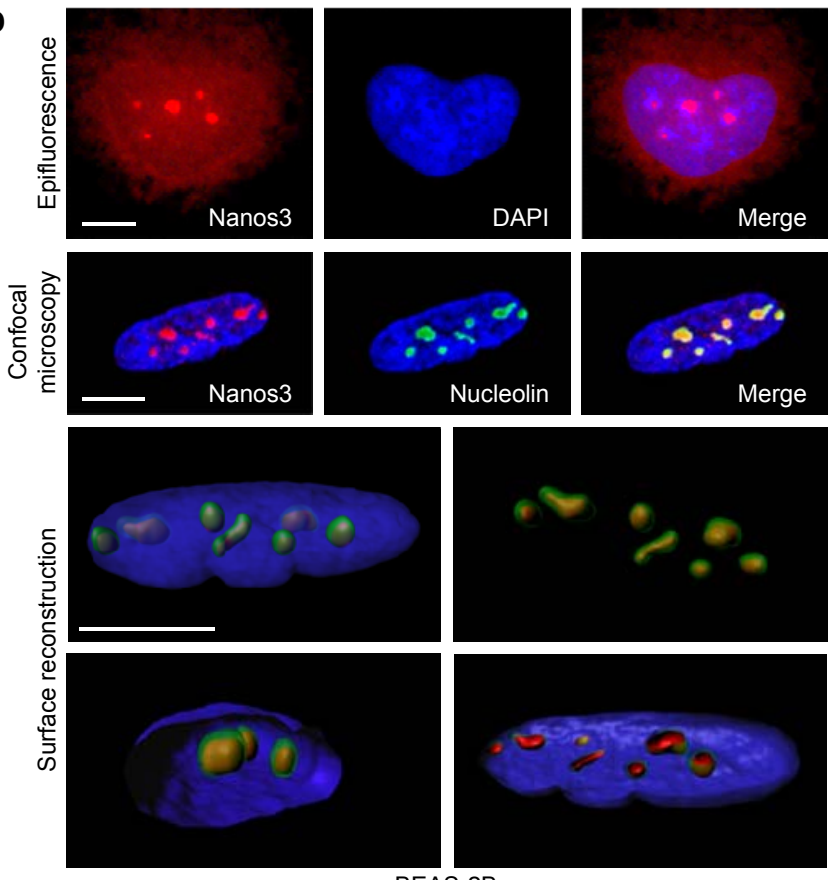

BEAS-2B 

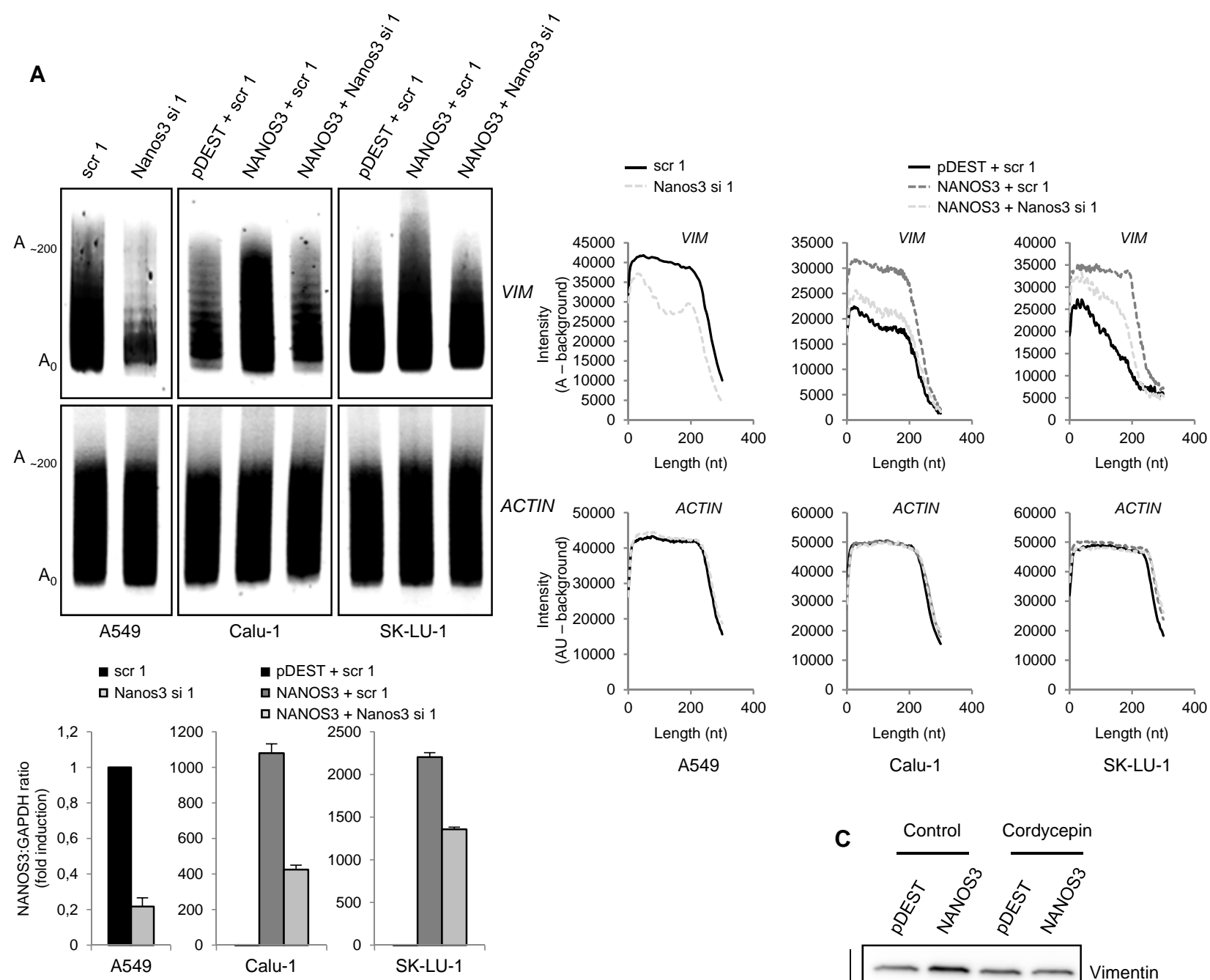

Calu-1

SK-LU-1

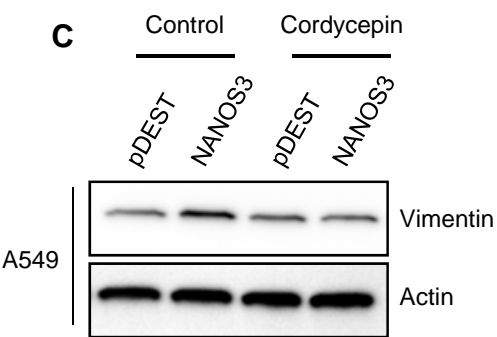

B

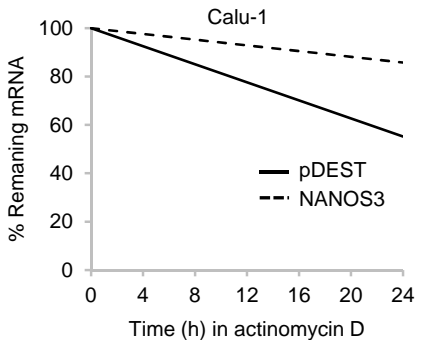

D

Hsa-miR-30a

VIM 3'UTR/WT VIM 3'UTR/Mut2 VIM 3'UTR/Mut12
Site 2 3'...CUA CAAAUGU...5' 3'...CUA SAte 1 IIIIII ...5' 3...CUA CAAAUG...5' 5...AAA GTTTAC AACATAATCTA GTTTAC AGA-3' 5....AAA GTCCCC AACATAATCTA GTTTAC AGA-3'
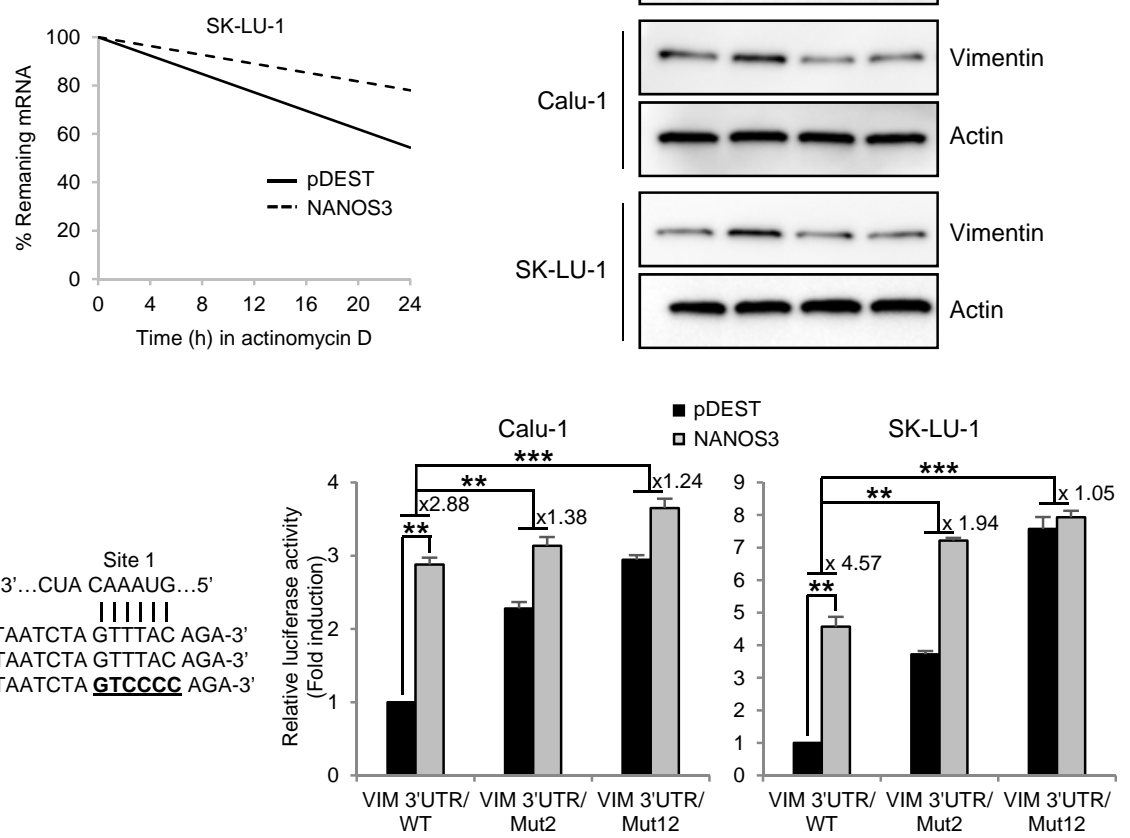

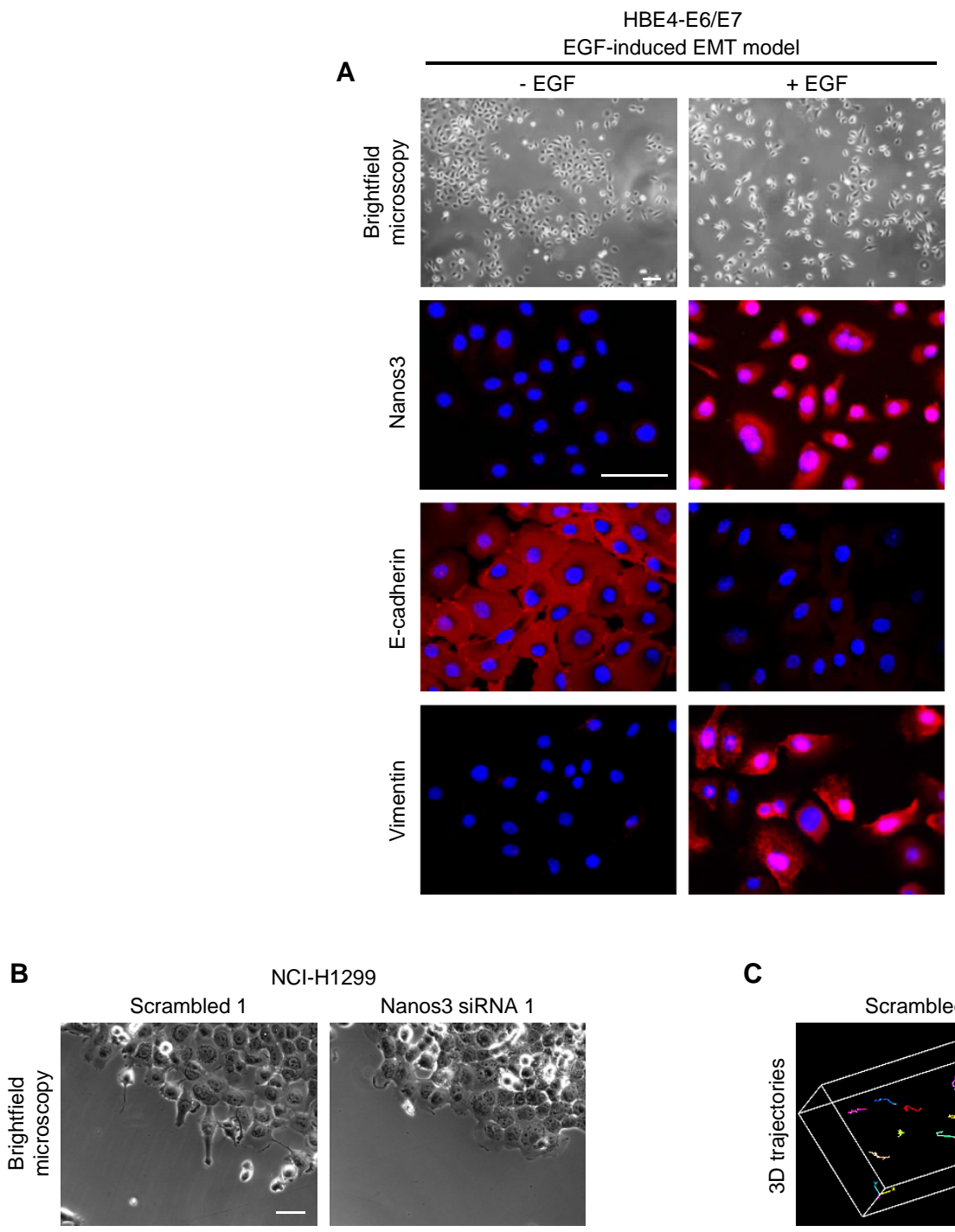

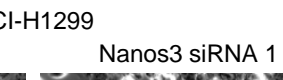

C
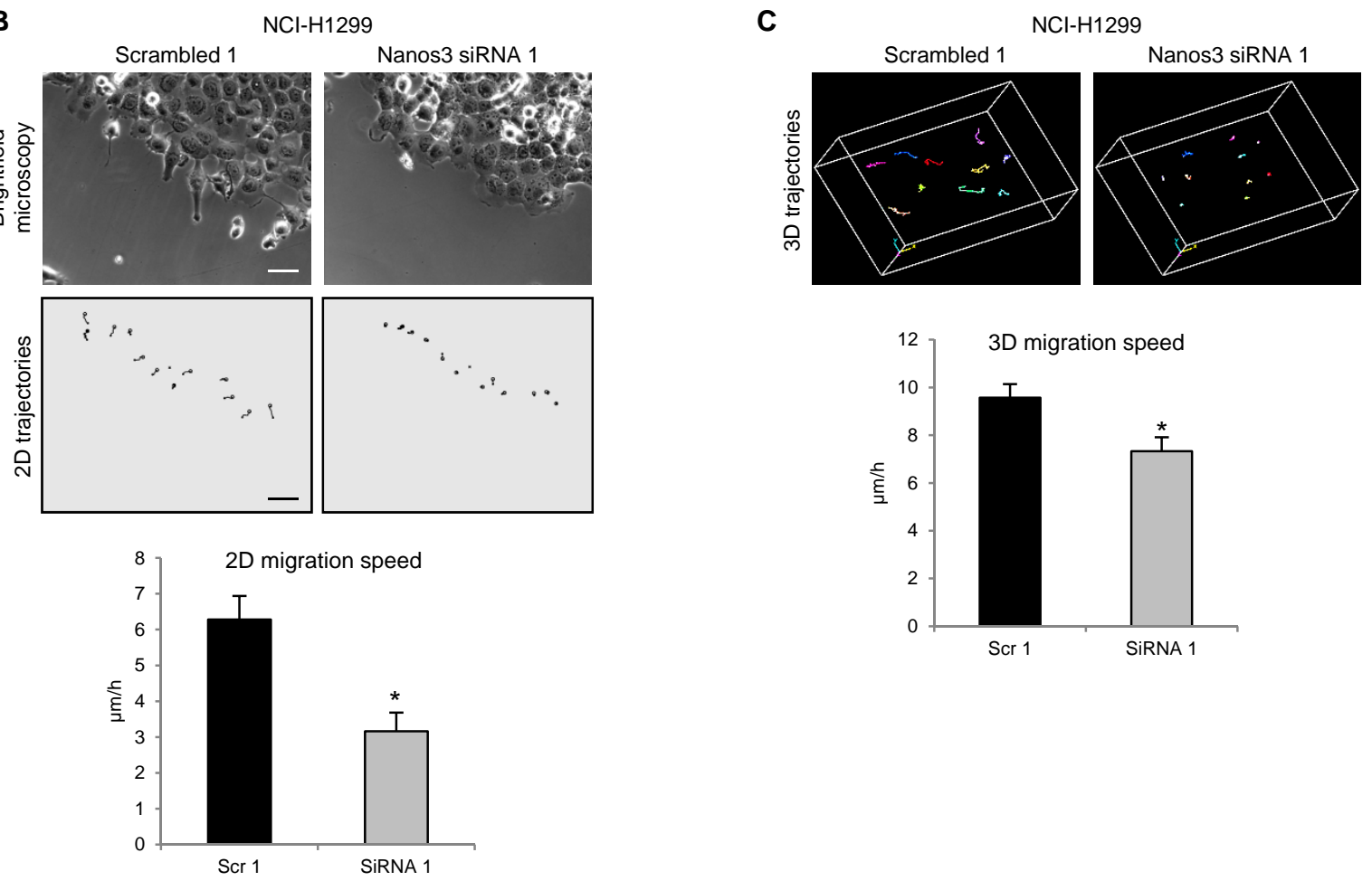
A

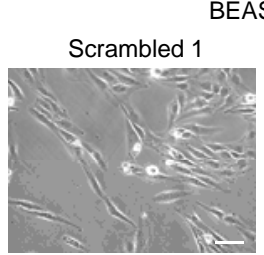

Scrambled 2

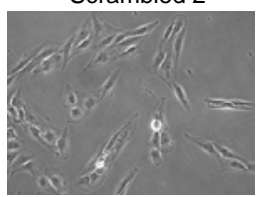



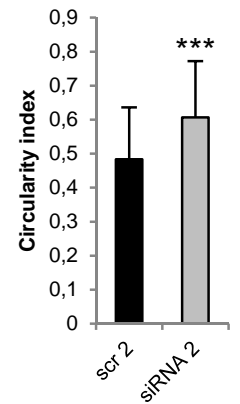

Nanos3 siRNA 2
B BEAS-2B
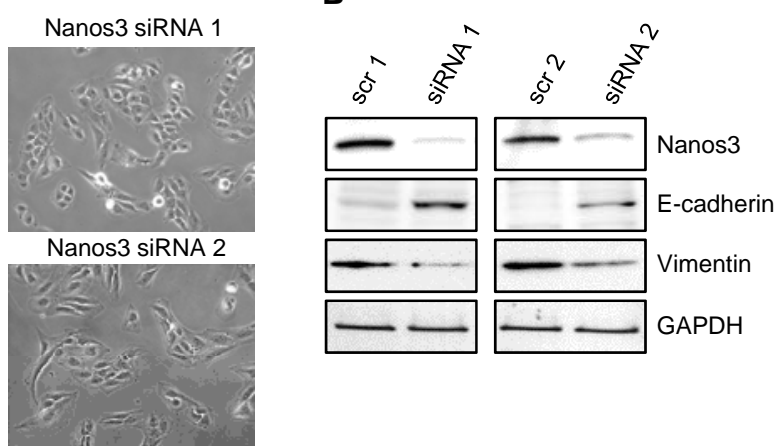
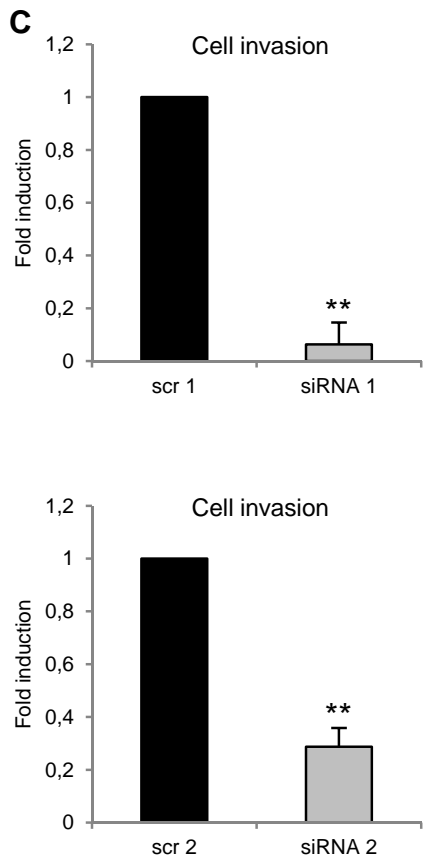
A

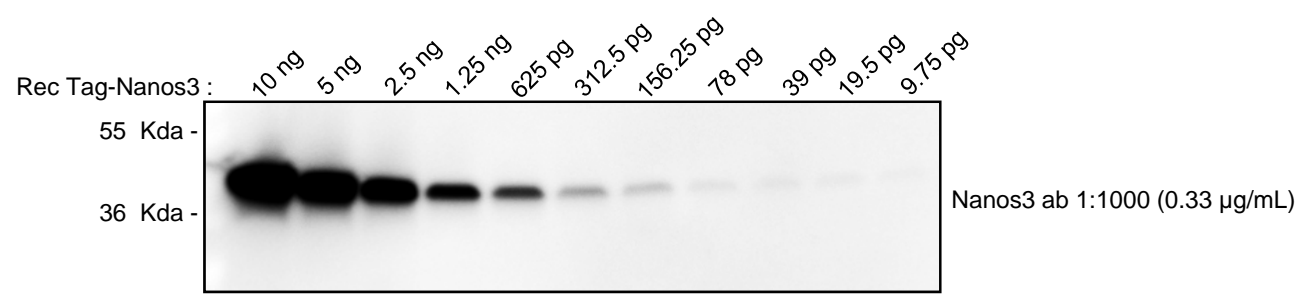

B

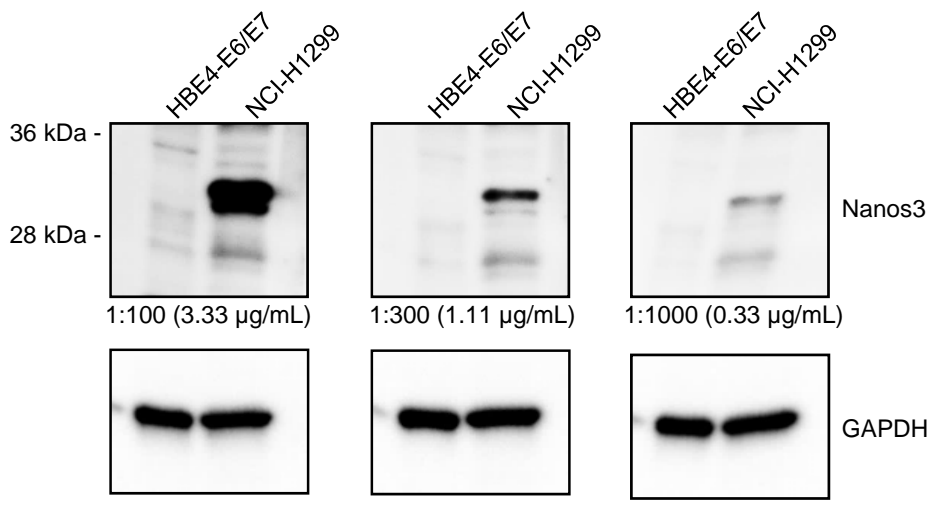

C

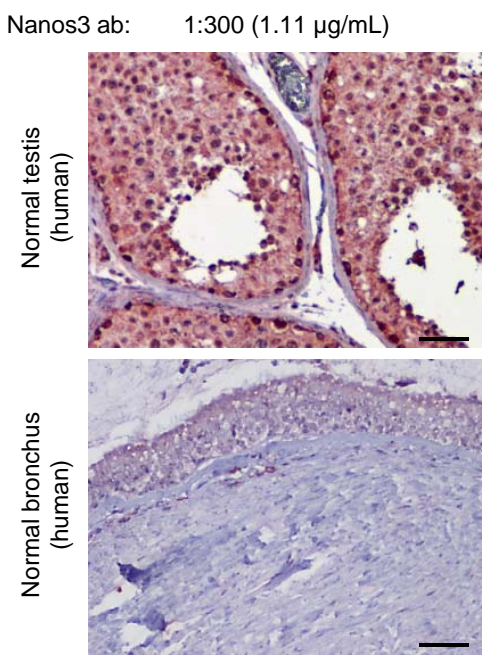

$1: 1000(0.33 \mu \mathrm{g} / \mathrm{mL})$

1:3000 $(0.11 \mu \mathrm{g} / \mathrm{mL})$

$1: 10000(0.03 \mu \mathrm{g} / \mathrm{mL})$
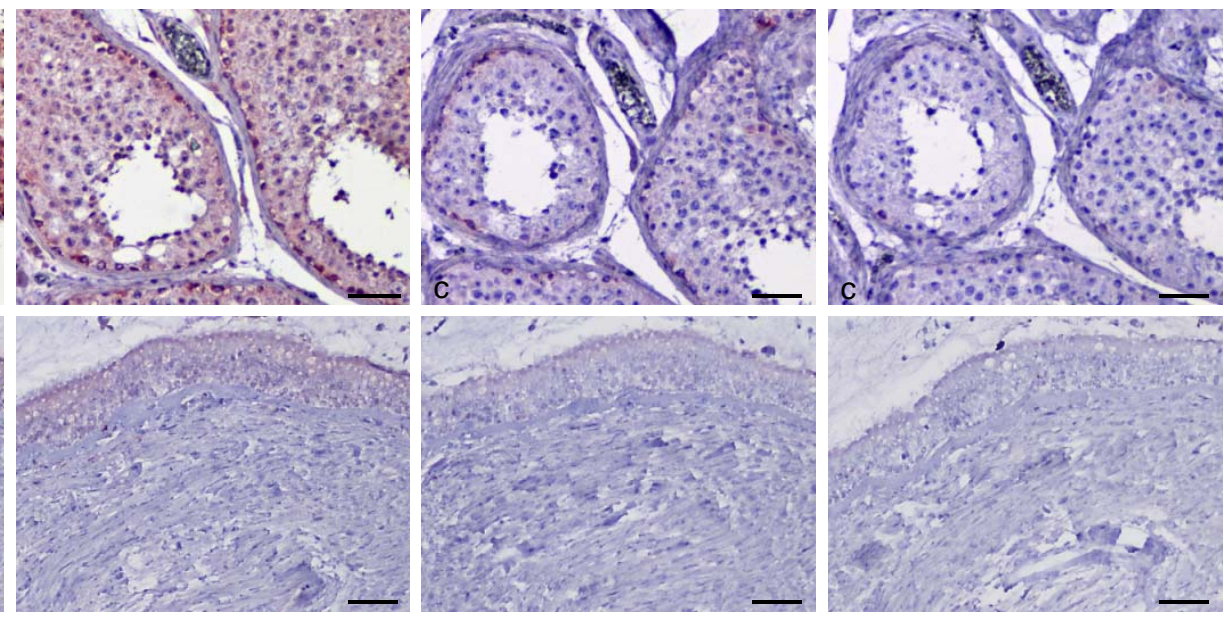


\section{Supplementary materials and methods}

\section{Cell culture}

All culture media and reagents were from Gibco (Invitrogen, Carlsbad, CA, USA). Cells used for experiments were passaged for fewer than six months after resuscitation. A549 and BEAS2B were cultured in DMEM containing 10\% fetal calf serum (FCS), NCI-H1299 in RPMI containing 10\% FCS, Calu-1 and SK-LU-1 in MEM containing 10\% FCS and supplemented with 1x MEM non-essential amino acids and $1 \mathrm{mM}$ sodium pyruvate, and HBE4-E6/E7 in Keratinocyte-SFM supplemented with $0.2 \mathrm{ng} / \mathrm{ml}$ EGF and $25 \mu \mathrm{g} / \mathrm{ml}$ bovine pituitary extract.

\section{Antibodies}

Antibodies used for western blotting (WB), immunohistochemistry (IHC), immunofluorescence (IF), and RNA immunoprecipitation were as follows: rabbit polyclonal antibodies to Nanos3 (WB, IHC and IF:1:1000; 21679-1-AP, Proteintech, Chicago, USA), and PARP (WB: 1:1000; Roche diagnostics GmbH, Mannheim, Germany); mouse monoclonal antibodies to Vimentin (WB: 1:1000, IHC: 1:600; IF: 1:25; clone V9; Dako, Glostrup, Denmark), E-cadherin (WB: 1:2500, IF: 1:100; clone 36, BD Bioscience, Franklin Lakes, NJ, USA; IHC: 1/50; clone NCH-38, Dako), Nucleolin (WB: 1:5000, IF: 1:2000; clone 4E2; Abcam, Cambridge, UK), $\alpha 1-\mathrm{Na}^{+}-\mathrm{K}^{+}$(WB: 1:5000; clone 464.6, Abcam) and GAPDH (WB: 1:75000; clone 6C5, Chemicon, Millipore, Billerica, MA, USA). Nanos3 antibody was characterized for its specificity and sensitivity in western blotting and immunohistochemistry (Supplementary Fig. S3). 


\section{Immunohistochemistry scoring}

The staining intensity was graded on a scale of $0-3$ ( 0 , no staining; 1 , low; 2 , medium; 3 , high) and the extent of staining on a scale of $0-4(0$, no staining; $1,<25 \% ; 2,25-50 \% ; 3,50-75 \%$; $4,>75 \%)$. The intensity score was multiplied by the extent score to generate both cytoplasmic and nuclear scores $(0-12)$, which were then combined to obtain the immunostaining score $(0-24)$.

\section{Real-time qRT-PCR for in vivo analysis}

Total RNA samples from 20 human tissues, included in the FirstChoice Human Total RNA survey panel (AM6000), were purchased from Life Technologies Europe (Ghent, Belgium). cDNA was prepared with the iScript cDNA synthesis kit according to the manufacturer's instructions (Bio-Rad). qPCR reactions were performed using the "SensiFast SYBR No-ROX kit (GC Biotech, Alphen aan den Rijn, The Netherlands)" kit as recommended by the manufacturer, in a LightCycler 480 Instrument (Roche Diagnostics). Gene expression levels were normalized using the geometric mean of the most stable reference genes [44]. Forward and reverse primers for NANOS3 are described in Supplementary Table S3.

\section{Second EMT-inducible model}

HBE4-E6/E7 cells sparsely seeded on coverslip coated with type-I collagen were treated with $20 \mathrm{ng} / \mathrm{mL}$ EGF for 5 days, fixed in methanol, and analysed by immunofluorescence.

\section{D migration assay}

Cell migration analysis in a type-I collagen gel was performed as previously described [21]. 


\section{Supplementary figure legends}

\section{Supplementary Figure S1: Nanos3 is overexpressed during EMT induced in vitro and its silencing decreases $2 \mathrm{D}$ and $3 \mathrm{D}$ migration.}

A, EMT was induced in the human bronchial cell line HBE4-E6/E7 by treatment for 3 days with $20 \mathrm{ng} / \mathrm{ml}$ EGF. Cells cultured on glass coverslips were subjected to immunofluorescence detection of Nanos3, E-cadherin and Vimentin. Nuclei were counterstained with DAPI. Bars, $50 \mu \mathrm{m}$. B, Comparison of trajectories and migration speeds $(\mu \mathrm{m} / \mathrm{h})$ of NCI-H1299 cells transfected with scrambled or Nanos3 siRNA 1 in a 2D migration assay. Scale bars, $20 \mu \mathrm{m}$; Error bars, S.D., $\mathrm{n}=3 . * \mathrm{P}<0.05 . \mathbf{C}$, Comparison of trajectories and migration speeds along the x-y-z plane of NCI-H1299 cells transfected with scrambled or Nanos3 siRNA 1 in a 3D migration assay in type-I collagen. Error bars, S.D., $\mathrm{n}=3 .{ }^{*} \mathrm{P}<0.05$.

\section{Supplementary Figure S2: Nanos3 silencing induces EMT-associated invasion.}

A, The circularity index of BEAS-2B transfected with scrambled (scr 1 and scr 2) or Nanos3 siRNA (siRNA 1 and 2) was determined in vitro from phase contrast photographs using the ImageJ software. Scale bar, $20 \mu \mathrm{m}$; Error bars, S.D., $\mathrm{n}=60 . * * * \mathrm{P}<0.001 . \mathrm{B}$, Western blot analysis of the effect of Nanos3-targeting siRNA 1 and siRNA 2 or scrambled siRNA scr 1 and scr 2 on E-cadherin and Vimentin protein levels in BEAS-2B cells. The levels of GAPDH served as loading controls. C, Analysis of the effect of inhibiting Nanos3 by siRNA 1 and siRNA 2 on the invasive capacity of BEAS-2B cells in a modified Boyden chamber invasion assay. Data are expressed as fold induction relative to the respective controls. Error bars, S.D., $\mathrm{n}=3 . * * \mathrm{P}<0.01$ 


\section{Supplementary Figure S3: Nanos3 antibody characterization.}

A, Western blot detection of Nanos3 on a range of $10 \mathrm{ng}$ to $9.75 \mathrm{pg}$ of a recombinant $\mathrm{N}$ terminally tagged Nanos3 protein fragment (ab153643, Abcam) using Nanos3 antibody (216791-AP, Proteintech, Chicago, USA) at a concentration of $0.33 \mu \mathrm{g} / \mathrm{ml}$. B, Western blot detection of Nanos3 in lysates of HBE4-E6/E7 and NCI-H1299 cells (20 $\mu \mathrm{g}$ protein/lane) found by RTPCR to be respectively negative and positive for NANOS3 expression. Antibody 21679-1-AP was used at a concentration of 3.33, 1.11 or $0.33 \mu \mathrm{g} / \mathrm{ml}$. The levels of GAPDH served as loading controls. C, Immunohistochemical detection of Nanos3 in sections of human normal testis incubated with Nanos 3 antibody $21679-1-\mathrm{AP}$ at a concentration of $1.11,0.33,0.11$ or 0.03 $\mu \mathrm{g} / \mathrm{ml}$. Nanos 3 is strongly and specifically expressed by germ cells, especially type-A spermatogonia. Human normal bronchus served as negative control. Scale bars, $50 \mu \mathrm{m}$.

\section{Supplementary video}

\section{Supplementary video S1: Nanos3 silencing decreases 2D migration.}

Comparison of migratory capacities of NCI-H1299 cells transfected with scrambled (left) or Nanos3 siRNA 1 (right) in a 2D migration assay. A phase-contrast image, observed at a 16-fold magnification, was recorded every $5 \mathrm{~min}$ for a total of $20 \mathrm{~h}$. Video duration: $12 \mathrm{~s}$.

\section{Supplementary reference}

44. Vandesompele J, De PK, Pattyn F, et al. Accurate normalization of real-time quantitative RT-PCR data by geometric averaging of multiple internal control genes. Genome Biol 2002; 3: RESEARCH0034. 


\section{Supplementary Table S1}

Description of the population studied by immunohistochemistry.

\begin{tabular}{|c|c|}
\hline Variables & $\mathrm{n}=94$ \\
\hline Age (years) & $61.0 \pm 9.4$ \\
\hline \multicolumn{2}{|l|}{ Sex } \\
\hline Male & $76(80.9 \%)$ \\
\hline Female & $18(19.1 \%)$ \\
\hline \multicolumn{2}{|l|}{ Histological type } \\
\hline Adenocarcinomas & $26(27.7 \%)$ \\
\hline Squamous cell carcinomas & $50(53.2 \%)$ \\
\hline Large cell carcinomas & $8(8.5 \%)$ \\
\hline Others & $10(10.6 \%)$ \\
\hline \multicolumn{2}{|l|}{ Differentiation status } \\
\hline Well & $28(29.8 \%)$ \\
\hline Mild & $27(28.7 \%)$ \\
\hline Poor & $39(41.5 \%)$ \\
\hline \multicolumn{2}{|l|}{ Tumour size } \\
\hline $\mathrm{T} 1$ & $8(8.5 \%)$ \\
\hline $\mathrm{T} 2$ & $66(70.2 \%)$ \\
\hline $\mathrm{T} 3$ & $12(12.8 \%)$ \\
\hline $\mathrm{T} 4$ & $6(6.4 \%)$ \\
\hline Unknown & $2(2.1 \%)$ \\
\hline \multicolumn{2}{|l|}{ Lymph node metastases } \\
\hline N0 & $50(53.2 \%)$ \\
\hline N1 & $24(25.5 \%)$ \\
\hline $\mathrm{N} 2$ & $18(19.1 \%)$ \\
\hline Unknown & $2(2.1 \%)$ \\
\hline \multicolumn{2}{|l|}{ Tumour stage } \\
\hline IA & $5(5.3 \%)$ \\
\hline IB & $28(29.8 \%)$ \\
\hline IIA & $26(27.7 \%)$ \\
\hline IIB & $10(10.6 \%)$ \\
\hline IIIA & $19(20.2 \%)$ \\
\hline IIIB & $3(3.2 \%)$ \\
\hline IV & $1(1.1 \%)$ \\
\hline Unknown & $2(2.1 \%)$ \\
\hline \multicolumn{2}{|l|}{ Nanos3 score } \\
\hline Score 0 & $5(5.3 \%)$ \\
\hline Score 1-4 & $22(23.4 \%)$ \\
\hline Score 5-9 & $31(33.0 \%)$ \\
\hline Score $10-15$ & $22(23.4 \%)$ \\
\hline Score 16-24 & $14(14.9 \%)$ \\
\hline
\end{tabular}




\section{Supplementary Table S2}

Predicted subcellular localization of Nanos3

\begin{tabular}{|c|c|c|}
\hline Method & Localization (\%) & Reliability \\
\hline $\mathrm{NNCN}$ & Nuclear & 0.94 \\
\hline CELLO & Nuclear & 0.79 \\
\hline Euk-mPLoc & Nuclear & \\
\hline \multirow[t]{2}{*}{ SubLoc (v1.0) } & Nuclear & $\mathrm{EA}=91 \%$ \\
\hline & & $\mathrm{RI}=4$ \\
\hline \multirow[t]{2}{*}{ ESLPred } & Nuclear & $\mathrm{EA}=53 \%$ \\
\hline & & $\mathrm{RI}=1$ \\
\hline \multirow[t]{4}{*}{ PSORT II } & Nuclear $(47.8 \%)$ & \\
\hline & Cytoplasmic (26.1\%) & 0.48 \\
\hline & Cytoskeletal (13.0\%) & \\
\hline & Mitochondrial (13.0\%) & \\
\hline \multirow[t]{3}{*}{ Yloc+ } & Nuclear $(84.5 \%)$ & \\
\hline & Cytoplasmic (15.1\%) & 0.81 \\
\hline & Other $(0.4 \%)$ & \\
\hline
\end{tabular}

RI, reliability index; EA, expected accuracy 


\section{Supplementary Table S3}

Sequences of primers and siRNA

\begin{tabular}{ccc}
\hline Primers for RIP analysis & \\
\hline VIM & Forward & 5'-GCGTGACGTACGTCAGCAATATGA-3' \\
& Reverse & 5'-GTTCCAGGGACTCATTGGTTCTT-3' \\
CDH1 & Forward & 5'-CCCATCAGCTGCCCAGAAAATGAA-3' \\
& Reverse & 5'-CTGTCACCTTCAGCCATCCTGTTT-3' \\
\hline \multicolumn{2}{c}{ Primers for real-time qRT-PCR } & \\
\hline NANOS3 & Forward & 5'-GCAAACACAACGGCGAGTC-3' \\
(In vivo $)$ & Reverse & 5'-CGGGTGGTGTGGCTGTAGA-3' \\
NANOS3 & Forward & 5'-ACAAGGCGAAGACACAGGAC-3' \\
(In vitro $)$ & Reverse & 5'-AGGTGGACATGGAGGGAGA-3' \\
GAPDH & Forward & 5'-ACCAGGTGGTCTCCTCTGAC-3' \\
& Reverse & 5'-TGCTGTAGCCAAATTCGTTG-3' \\
VIM & Forward & 5'-GCGTGACGTACGTCAGCAATATGA-3' \\
& Reverse & 5'-GTTCCAGGGACTCATTGGTTCCTT-3' \\
CDH1 & Forward & 5'-GACACATTTATGGAACAGAAAATAACA-3' \\
& Reverse & 5'-AGTGGAAATGGCACCAGTGT-3' \\
18S & Forward & 5'-TTG-GTG-GAG-CGA-TTT-GTC-TG-3', \\
& Reverse & 5'-ATC-TCG-GGT-GGC-TGA-ACG-3' \\
\hline siRNA & & \\
\hline NANOS3 & Sil & 5'-GGAUCAGAAGCGCAGCCUG-3' \\
\hline Primer for mRNA poly(A) tail length analysis \\
\hline VIM & Forward & 5'-GGAGCGCAAGATAGATTTGG-3'
\end{tabular}

